\title{
Cyclophilin a signaling induces pericyte- associated blood-brain barrier disruption after subarachnoid hemorrhage
}

Pengyu Pan ${ }^{1,2}$, Hengli Zhao ${ }^{1}$, Xuan Zhang ${ }^{1}$, Qiang Li ${ }^{1}$, Jie Qu' ${ }^{1}$, Shilun Zuo ${ }^{1}$, Fan Yang ${ }^{3}$, Guobiao Liang ${ }^{2}$, John H. Zhang ${ }^{4}$, Xin Liu', Haiyang He ${ }^{5}$, Hua Feng ${ }^{1,6,7^{*}}$ and Yujie Chen ${ }^{1,7^{*}}$ (D)

\begin{abstract}
Objective: The potential roles and mechanisms of pericytes in maintaining blood-brain barrier (BBB) integrity, which would be helpful for the development of therapeutic strategies for subarachnoid hemorrhage (SAH), remain unclear. We sought to provide evidence on the potential role of pericytes in BBB disruption and possible involvement and mechanism of CypA signaling in both cultured pericytes and SAH models.

Methods: Three hundred fifty-three adult male C57B6J mice weighing 22 to $30 \mathrm{~g}, 29 \mathrm{CypA}^{-/-}$mice, $30 \mathrm{CypA}^{+/+}$ (flox/flox) mice, and 30 male neonatal C57B6J mice were used to investigate the time course of CypA expression in pericytes after SAH, the intrinsic function and mechanism of CypA in pericytes, and whether the known receptor CD147 mediates these effects.

Results: Our data demonstrated both intracellular CypA and CypA secretion increased after SAH and could activate CD147 receptor and downstream NF-KB pathway to induce MMP9 expression and proteolytic functions for degradation of endothelium tight junction proteins and basal membranes. CypA served as autocrine or paracrine ligand for its receptor, CD147. Although CypA could be endocytosed by pericytes, specific endocytosis inhibitor chlorpromazine did not have any effect on MMP9 activation. However, specific knockdown of CD147 could reverse the harmful effects of CypA expression in pericytes on the BBB integrity after SAH.

Conclusions: This study demonstrated for the first time that CypA mediated the harmful effects of pericytes on BBB disruption after SAH, which potentially mediated by CD147/NF-KB/MMP9 signal, and junction protein degradation in the brain. By targeting CypA and pericytes, this study may provide new insights on the management of SAH patients.
\end{abstract}

Keywords: Subarachnoid Hemorrhage, Pericyte, Blood-brain barrier, Cyclophilin A, CD147

\section{Introduction}

Despite years of research, early brain injury, which is the main contributor to the mortality and poor prognosis of patients after subarachnoid hemorrhage (SAH), remains a worrisome complication of ruptured intracranial aneurysms [1]. The pathophysiology of SAH and other stroke events involves changes in the cerebral vascular neural network, including the arterial and venous systems as well as neuronal cells, other support cells, and cellular

\footnotetext{
*Correspondence: fenghua8888@vip.163.com; yujiechen6886@foxmail.com ${ }^{1}$ Department of Neurosurgery, Southwest Hospital, Army Medical University (Third Military Medical University), Chongqing 400038, China Full list of author information is available at the end of the article
}

matrices [2]. Within this vascular neural network, blood-brain barrier damage contributes to vasogenic brain edema, which is one of the most common pathophysiological changes after SAH and has become a focus of interest in the development of novel therapeutic strategies for SAH patients. However, the exact mechanism underlying blood-brain barrier disruption and remodeling has not been clarified.

Pericytes are partially sandwiched between endothelial cells and astrocyte end feet and represent important constituents of the neurovascular unit, and they have traditionally been considered essential for normal blood-brain barrier function [3]. A previous study indicated that

(C) The Author(s). 2020 Open Access This article is distributed under the terms of the Creative Commons Attribution 4.0 International License (http://creativecommons.org/licenses/by/4.0/), which permits unrestricted use, distribution, and 
pericytes bidirectionally control microvessel diameters and regulate cerebral blood flow [4]. Our previous study further demonstrated that pericyte $\alpha$-SMA phenotype transformation caused acute microvessel/pearl-like constriction and neurological deficits in the setting of SAH [5, 6]. However, a recent study suggested that pericytes might have a negative influence on neurovascular integrity and cause neuronal degeneration in apolipoprotein E-deficient mice [7]. More direct evidence was found using a two-photon microscope, which showed that pericytes contribute to rapid and localized proteolytic degradation around pericyte somata during ischemia [8].

Therefore, as a multifunctional cell type, the pathophysiological changes in pericytes after SAH should be further investigated [9]. Cyclophilin A (СурA), which is encoded by the peptidylprolyl isomerase A gene, is a small molecule protein located in the cytoplasm and the extracellular space, and it has been widely used as a target of immunosuppressant after organ transplantation for anti-rejection purposes as well as in the treatment of autoimmune diseases [10]. Our previous work also indicate that CypA-specific inhibitor, Cyclosporine A, could alleviate MMP9-associated blood-brain barrier disruption [11]. Recent studies have reported that CypA in pericytes is actually the core of blood-brain barrier integrity in neurodegenerative diseases [7]. However, the potential roles of pericytes in maintaining the integrity of the blood-brain barrier following SAH and the exact mechanisms of this process, which would be helpful for the development of therapeutic strategies, remain unclear. In the present study, we sought to provide additional evidence on the potential role of pericytes in bloodbrain barrier disruption and the possible involvement and mechanism of CypA signaling in both cultured primary pericytes and SAH models.

\section{Materials and methods \\ Animals}

Three hundred fifty-three adult male C57B6J mice weighing 22 to $30 \mathrm{~g}$, 29 CypA knockout $\left(\mathrm{CypA}^{-1-}\right)$ mice, $30 \mathrm{CypA}^{+/+}$(flox/flox) mice, and 30 male neonatal C57B6J mice were provided by the Experimental Animal Center of the Third Military Medical University (Chongqing, China) for the present study. CypA ${ }^{-/-}$mice were purchased form The Jackson Laboratory (ME, USA) and backcrossing at least ten generations into C57B6J background. All experimental procedures were approved by the Ethics Committee of Southwest Hospital and performed in accordance with the guidelines in the National Institutes of Health Guide for the Care and Use of Laboratory Animals and followed the ARRIVE guidelines.

\section{Experimental design}

The present study contained four experiments (Additional file 1: Figure S1), which were designed as follows.

\section{Experiment I}

To determine the time course of CypA expression in pericytes after $\mathrm{SAH}, 44$ mice were randomly assigned into seven groups: Sham $(n=7)$, SAH $3 \mathrm{~h}(n=6)$, SAH $6 \mathrm{~h}(n=$ $6)$, SAH $12 \mathrm{~h}(n=6)$, SAH $24 \mathrm{~h}(n=7)$, SAH $48 \mathrm{~h}(n=6)$, and SAH $72 \mathrm{~h}(n=6)$. Western blots were used to detect the CypA protein expression in microvessels isolated from the ipsilateral/left hemisphere in each group. Immunohistochemical staining of CypA, PDGFR $\beta / C D 13$, and Lectin was performed $24 \mathrm{~h}$ after $\mathrm{SAH}$ induction to confirm the spatial distribution of CypA in the pericytes $(n=2)$. None of the sham-operated mice died, and eight mice died within $72 \mathrm{~h}$ and after SAH caused by severe hemorrhagic volume.

\section{Experiment II}

To define the intrinsic function of CypA in the pericytes, 30 $\mathrm{CypA}^{+/+}$(flox/flox) adult C57B6) mice and $29 \mathrm{CypA}^{-/-}$mice were randomly assigned into four groups: flox/flox + Sham $(n=13)$, flox/flox + SAH $(n=13), \mathrm{KO}+\operatorname{Sham}(n=13)$, and $\mathrm{KO}+\mathrm{SAH}(n=13)$ groups. Then, modified Garcia tests and beam balance tests were performed $24 \mathrm{~h}$ after SAH induction to evaluate the neurological deficits in each group $(n=6)$. In addition, an Evans blue extravasation assessment and fluorescence imaging of Evans blue and Cadaverine extravasation $(n=6)$ were performed $24 \mathrm{~h}$ after SAH induction to detect the blood-brain barrier disruptions. Immunohistochemical staining was also performed to detect the spatial expression of collagen IV and Lectin in the ipsilateral/left hemisphere $24 \mathrm{~h}$ after SAH induction $(n=2)$. None of the sham-operated mice died, and three $\mathrm{CypA}^{+/+}$(flox/ flox) mice and two $\mathrm{CypA}^{-/-}$mice died after SAH caused by severe hemorrhagic volume.

Furthermore, 155 wild-type adult C57B6J mice were randomly divided into the following groups: Sham $(n=31)$; $\mathrm{SAH}+$ vehicle $(2 \mu \mathrm{l}$ of sterile saline; $n=31), \mathrm{SAH}+\mathrm{CypA}$ (200 ng in $2 \mu \mathrm{l}$ of sterile saline; $n=31$ ); SAH + scrambled small interfering RNA (SAH + Scr siRNA; 500 pmol in a 2- $\mu \mathrm{l}$ mixture of 1:1 DEPC-treated water and liposome; $n=31$ ); and SAH + CypA small interfering RNA (SAH + CypA siRNA; RiboBio, Guangzhou, China; 500 pmol in a 2- $\mu$ l mixture of 1:1 DEPC-treated water and liposome; $n=31$ ). Scrambled siRNA or CypA siRNA was intracerebroventricularly injected at $48 \mathrm{~h}$ before SAH. Modified Garcia tests $(n=6)$, beam balance tests $(n=$ $6)$, brain water content assessment $(n=6)$, and Evans blue extravasation assessment $(n=6)$ were performed $24 \mathrm{~h}$ after SAH induction. Immunohistochemical staining was also performed to detect the spatial expression of collagen IV and lectin in the ipsilateral/left hemisphere $24 \mathrm{~h}$ after $\mathrm{SAH}$ induction $(n=1)$. Western blots were performed to detect the P-p65 and MMP9 protein expression in microvessels isolated from the ipsilateral/ left hemisphere of each group $(n=6)$; and the ZO-1, collagen IV, Occludin, and claudin 5 expression in the 
total cortex protein $(n=6)$. In addition, gelatin zymography $(n=6)$ was used to detect the proteolytic capacity of MMP9. None of the sham-operated mice died, and 63 mice died within $72 \mathrm{~h}$ and after SAH caused by severe hemorrhagic volume.

\section{Experiment III}

To investigate the secretion of CypA from the pericytes after $\mathrm{SAH}, 30$ neonatal mice were used for the primary pericyte culture. To determine the time course of the intracellular and extracellular CypA expression in the pericytes after oxyhemoglobin $(\mathrm{Hb})$ stimulation, cultured primary pericytes were randomly assigned into seven groups: Vehicle $(n=6), \mathrm{Hb} 3 \mathrm{~h}(n=6), \mathrm{Hb} 6 \mathrm{~h}(n=6)$, $\mathrm{Hb} 12 \mathrm{~h}(n=6), \mathrm{Hb} 24 \mathrm{~h}(n=6)$, Hb $48 \mathrm{~h}(n=6)$, and $\mathrm{Hb} 72 \mathrm{~h}(n=6)$. Western blots were performed to detect the protein expression of intracellular CypA in the pericytes of each group, and the results were confirmed via immunohistochemical staining at $24 \mathrm{~h}$ after $\mathrm{Hb}$ stimulation. The supernatants of the culture media were collected to measure the secreted CypA via ELISA.

To determine whether endocytosis of CypA occurs in the pericytes and to screen for the inhibitors of CypA endocytosis, exogenous CypA was conjugated with FITC to investigate the endocytosis of CypA in the cultured pericytes, and laser confocal scanning of CypA-FITC was performed in the cultured primary pericytes at $10 \mathrm{~min}, 30$ min, $1 \mathrm{~h}, 2 \mathrm{~h}$, and $3 \mathrm{~h}$ after adding CypA-FITC $(1 \mu \mathrm{g} / \mathrm{ml}$, Novoprotein, Shanghai, China) to the culture media. In addition, the cultured primary pericytes were randomly assigned to seven groups: DMSO $(n=6)$; DMSO + CypAFITC $(1 \mu \mathrm{g} / \mathrm{ml})(n=6)$; Amiloride hydrochloride $(100 \mu \mathrm{M}$, Sigma-Aldrich, Shanghai, China) + CypA-FITC $(1 \mu \mathrm{g} / \mathrm{ml})$ $(n=6)$; Dynasore $(80 \mu M$, Sigma-Aldrich, Shanghai, China) + CypA-FITC $(1 \mu \mathrm{g} / \mathrm{ml})(n=6)$; Genistein $(200 \mu \mathrm{M}$, Sigma-Aldrich, Shanghai, China) + CypA-FITC $(1 \mu \mathrm{g} / \mathrm{ml})$ $(n=6)$; Chloroquine diphosphate $(200 \mu \mathrm{M}$, Sigma-Aldrich, Shanghai, China $)+$ CypA-FITC $(1 \mu \mathrm{g} / \mathrm{ml})(n=6)$; and Chlorpromazine hydrochloride (30 $\mu \mathrm{M}$, Sigma-Aldrich, Shanghai, China $)+$ CypA-FITC $(1 \mu \mathrm{g} / \mathrm{ml})(n=6)$. Flow cytometry was used to detect the FITC-positive cells percentage in each group.

To determine whether secretion inhibitor Exo1 and Exo2 reducing CypA secretion from pericytes, cultured primary pericytes were randomly assigned to five groups: DMSO $(n=6) ; \mathrm{Hb}(n=6) ; \mathrm{Hb}+$ vehicle $(n=6) ; \mathrm{Hb}+$ Exo1 $(100 \mu M$, Sigma-Aldrich, Shanghai, China) $(n=6)$; and $\mathrm{Hb}+$ Exo2 $(50 \mu \mathrm{M}$, Sigma-Aldrich, Shanghai, China) $(n=6)$. Secreted CypA and MMP9 in the medium were detected by ELISA.

\section{Experiment IV}

To determine whether the known receptor CD147 mediates the effects of CypA, the following experiments were performed. First, to determine the time course of CD147 expression in pericytes after $\mathrm{SAH}, 44$ mice were randomly assigned into seven groups: Sham $(n=7)$, SAH 3 $\mathrm{h}(n=6)$, SAH $6 \mathrm{~h}(n=6)$, SAH $12 \mathrm{~h}(n=6)$, SAH $24 \mathrm{~h}$ $(n=7)$, SAH $48 \mathrm{~h}(n=6)$, and SAH $72 \mathrm{~h}(n=6)$. Western blots were performed to detect the CD147 protein expression in microvessels isolated from the ipsilateral/ left hemisphere of each group. Immunohistochemical staining of CD147, PDGFR $\beta / C D 13$, and Lectin was performed $24 \mathrm{~h}$ after SAH induction to confirm the spatial distribution of CD147 in the pericytes $(n=1)$. In addition, cultured primary pericytes were randomly assigned into seven groups: Vehicle $(n=6), \mathrm{Hb} 3 \mathrm{~h}(n=$ 6), Hb 6 h $(n=6)$, Hb 12 h $(n=6)$, Hb $24 \mathrm{~h}(n=6), \mathrm{Hb}$ $48 \mathrm{~h}(n=6)$, and $\mathrm{Hb} 72 \mathrm{~h}(n=6)$. Western blots were performed to detect the protein expression level of intracellular CD147 in the pericytes of each group, and the results were confirmed via immunohistochemical staining $24 \mathrm{~h}$ after $\mathrm{Hb}$ stimulation. All mice and cultured pericytes specimen in experiment III were from experiment I.

Second, to determine whether CD147 play a role in endocytosis of CypA by pericyte, cultured primary pericytes were randomly assigned into four groups: DMSO $(n=6) ;$ DMSO + CypA-FITC $(1 \mu \mathrm{g} / \mathrm{ml})(n=6)$; CypA-FITC $(1 \mu \mathrm{g} / \mathrm{ml})+$ scrambled siRNA; and CypAFITC $(1 \mu \mathrm{g} / \mathrm{ml})+$ CD147 siRNA (RiboBio, Guangzhou, China). Flow cytometry was used to detect the FITCpositive cells percentage in each group. Then, to detect MMP9 secretion, cultured pericytes were randomly assigned into nine groups: DMSO; $\mathrm{Hb}(10 \mu \mathrm{M})+$ DMSO; $\mathrm{Hb}+$ CypA $(100 \mathrm{ng} / \mathrm{ml}) ; \mathrm{Hb}+$ scrambled siRNA; $\mathrm{Hb}+$ CypA siRNA; $\mathrm{Hb}+$ CypA + scrambled siRNA; $\mathrm{Hb}+$ CypA + CD147 siRNA; Hb + CypA + DMSO; and Hb + CypA + chlorpromazine $(30 \mu \mathrm{M})$. An ELISA was performed to measure the level of MMP9 in the supernatant of the culture media, and gelatin zymography was used to detect its proteolytic capacity.

Third, to validate the role of CD147 after SAH in vivo, 125 mice were randomly assigned into five groups: Sham $(n=25) ; \mathrm{SAH}+$ vehicle $(2 \mu \mathrm{l}$ of sterile saline; $n=25)$; SAH + CypA (200 ng in $2 \mu \mathrm{L}$ of sterile saline; $n=25$ ); $\mathrm{SAH}+\mathrm{CypA}$ (200 ng in $2 \mu \mathrm{l}$ of sterile saline) + scrambled siRNA (500 pmol in a 2- $\mu$ l mixture of 1:1 DEPCtreated water and liposome; $n=25)$; and SAH + CypA (200 ng in 2- $\mu$ l sterile saline) + CD147 siRNA (500 pmol in a 2- $\mu$ l mixture of 1:1 DEPC-treated water and liposome; $n=25$ ). Scrambled siRNA or CypA siRNA was intracerebroventricularly injected $48 \mathrm{~h}$ before SAH. Modified Garcia tests $(n=6)$, beam balance tests $(n=$ $6)$, brain water content assessments $(n=6)$, and Evans blue extravasation assessments $(n=6)$ were performed $24 \mathrm{~h}$ after SAH induction. Immunohistochemical staining was also performed to detect the spatial expression of collagen IV and Lectin in the ipsilateral/left 
hemisphere $24 \mathrm{~h}$ after SAH induction $(n=1)$. Western blots $(n=6)$ were performed to detect the P-p65 and MMP9 protein expression in microvessels isolated from the ipsilateral/left hemisphere of each group; and ZO-1, collagen IV, Occludin, and claudin 5 expression was detected in the total cortex protein. In addition, gelatin zymography $(n=6)$ was used to detect the proteolytic capacity of MMP9. None of the sham-operated mice died, and 28 mice died within $72 \mathrm{~h}$ and after SAH caused by severe hemorrhagic volume.

\section{SAH model}

The SAH mouse model was generated by endovascular perforation as previously described [12]. Briefly, under sodium pentobarbital anesthesia $(40 \mathrm{mg} / \mathrm{kg}$, intraperitoneally injected), the external carotid artery was identified and distally transected into a 2-mm stump. A 5-0 sharpened monofilament nylon suture was advanced into the internal carotid artery through the external carotid artery until resistance was felt, and then it was pushed $2 \mathrm{~mm}$ further to penetrate the bifurcation of the anterior and middle cerebral artery. The suture was then withdrawn, and the internal carotid artery was reperfused to produce the SAH. Sham-operated mice underwent the same procedure, although the suture was withdrawn without perforating the cerebral artery after feeling resistance.

\section{Intracerebroventricular injection}

The intracerebroventricular injection procedure was performed as previously described [13]. A small burr hole was drilled $1.0 \mathrm{~mm}$ lateral to the bregma on the skull. The needle of a 10- $\mu$ l Hamilton syringe (Microliter 701; Hamilton Company, Reno, NV, USA) was stereotactically inserted into the left lateral ventricle through the burr hole $3.0 \mathrm{~mm}$ below the horizontal plane of the bregma. A $2 \mu \mathrm{l}$ volume of CypA (Novoprotein, Shanghai, China) in sterile saline was infused at a rate of $0.2 \mu \mathrm{l} /$ min $1 \mathrm{~h}$ after SAH induction, whereas $500 \mathrm{pmol} / 2 \mu \mathrm{l}$ of CypA, CD147, or scrambled siRNA was infused at the same rate $48 \mathrm{~h}$ before SAH modeling. The syringe was left in situ for an additional $10 \mathrm{~min}$ before slowly removing it from the animal.

CypA siRNA and CAD147 siRNA are pools of three different siRNA duplexes in order to improve the knockdown efficiency.

Targeting sequences of CypA siRNA are provided in $5^{\prime} \rightarrow 3^{\prime}$ orientation as follows:

\section{(I) TGACTTTACACGCCATAAT \\ (II) CCATCTACGGAGAGAAATT \\ (III)GCATCTTGTCCATGGCAAA}

Targeting sequences of CD147 siRNA are provided in $5^{\prime} \rightarrow 3^{\prime}$ orientation as follows:
(I) GGATCAAGGTCGGAAAGAA

(II) GCAAGTCCGATGCATCCTA

(III)TCAGCAACCTTGACGTAAA

\section{Neurological outcome assessment}

Neurological deficits were evaluated 24 and $72 \mathrm{~h}$ after $\mathrm{SAH}$ induction using the modified Garcia scale, which is an 18-point score system, and the beam balance test, which is a 5-point score system, in the outcome study [13]. The modified Garcia assessment consisted of six tests covering spontaneous activity, spontaneous movement of four limbs, forepaw outstretching, climbing, body proprioception, and response to whisker stimulation (3-18 points). For the beam balance test, the mice were placed on a beam and their walking distance within 1 min (0-5 points) was observed. The mean of the neurological score was evaluated by two blinded observers for grading.

\section{SAH grade assessment}

An 18-point SAH severity grading system was used as previously described [14]. The basal cistern was divided into six segments that could be scored from 0 to 3 according to the amount of subarachnoid blood clotting. The total score was calculated by adding the scores from six segments $(0-18$ points). Animals that received a score $<8$ were excluded from the study.

\section{Brain water content}

The brains were quickly separated into left and right cerebral hemispheres, cerebellum, and brain stem and then weighed (wet weight). Then, the brain samples were dried in an oven at $55{ }^{\circ} \mathrm{C}$ for $48 \mathrm{~h}$ and weighed again (dry weight). The percentage water content was calculated as follows: ([wet weight - dry weight]/wet weight) $\times 100 \%$.

\section{Extravasation and fluorescence of Evans blue and Cadaverine-Alexa Flour $\mathbf{5 5 5}$}

Evans blue extravasation was performed as previously described [6]. At $24 \mathrm{~h}$ post-operation, Evans blue dye ( $2 \%, 5 \mathrm{ml} / \mathrm{kg}$; Sigma-Aldrich, St. Louis, MO, USA) or Cadaverine-Alexa Flour $555(500 \mu \mathrm{g} / \mathrm{kg}$; Thermo Fisher Scientific, Waltham, MA, USA) was injected and administered $>2$ min into the left femoral vein and allowed to circulate for $60 \mathrm{~min}$. Under anesthesia, the mice were euthanized by an intracardial perfusion of phosphatebuffered saline (PBS). Then fluorescent efficiency of the Evans blue and Cadaverine extravasation was measured by a living image system, the IVIS Spectrum CT (PerkinElmer, Waltham, MA, USA) equipped with fluorescent filter sets (Evans blue, excitation/emission, 470/680 nm; Cadaverine-Alexa Flour 555, excitation/emission, 550/ $570 \mathrm{~nm}$ ) and average efficiency of Cadaverine-Alexa 
Flour 555 was calculated by IVIS living image 4.5 software (PerkinElmer, Waltham, MA, USA). Subsequently, the brains were removed and divided into left and right cerebral hemispheres, and then the brain samples were weighed, homogenized in saline, and centrifuged at 15,000 $\mathrm{g}$ for $30 \mathrm{~min}$. Next, an equal volume of trichloroacetic acid was added to the resultant supernatant. The samples were then incubated overnight at $4{ }^{\circ} \mathrm{C}$ and centrifuged at 15 , $000 \mathrm{~g}$ for $30 \mathrm{~min}$. The resultant supernatant was then spectrophotometrically quantified for the extravasated Evans blue dye at $610 \mathrm{~nm}$.

\section{Isolation of microvessels}

Microvessels were isolated using dextran gradient centrifugation followed by sequential cell-strainer filtrations as previously described [15]. First, the mouse brain was isolated, and the meninges were removed in ice-cold PBS containing $2 \%$ fetal bovine serum (FBS). The cortex was collected, and all macroscopically visible white matter was removed. The cortex was then homogenized in FBS with a glass dounce homogenizer. Added Dextran (70 $\mathrm{kDa}$, Sigma-Aldrich, Shanghai, China) into the samples to yield a final concentration of $16 \%$, and the samples were thoroughly mixed sequentially. The samples were then centrifuged at $6000 \mathrm{~g}$ for $15 \mathrm{~min}$. The microvessel pellet located at the bottom of the tubes was collected and filtered through $100 \mathrm{~m}$ and $45 \mathrm{~m}$ cell strainers (BD, Franklin Lakes, NJ, USA). The microvessels that remained on top of the $45 \mathrm{~m}$ cell strainer were collected in PBS for further analysis.

\section{Western blot}

Western blot assays were performed as previously described $[12,16]$. The protein extracted from the left hemisphere (perforation side) was used for the Western blot analysis. Equal amounts of total protein $(30 \mu \mathrm{g})$ were loaded in each lane of SDS-PAGE gels. After gel electrophoresis, the protein was transferred onto a nitrocellulose membrane, which was then blocked via blocking buffer for $2 \mathrm{~h}$ at room temperature. The following primary antibodies were diluted to incubate with the membrane under gentle agitation at $4{ }^{\circ} \mathrm{C}$ overnight: anti-CypA (1:500; Abcam, Cambridge, UK), anti-CD147 (1:500; Abcam, Cambridge, UK), collagen IV (1:500; Abcam, Cambridge, UK), anti-ZO-1 (1:500; Invitrogen, Waltham, MA, USA), antiOccludin (1:500; Invitrogen, Waltham, MA, USA), claudin 5 (1:500; Thermo Fisher Scientific, Waltham, MA, USA), Pp65 (1:500; Abcam, Cambridge, UK), p65 (1:500; Abcam, Cambridge, UK), and MMP9 (1:500; Abcam, Cambridge, UK). GAPDH and $\beta$-actin were used as internal loading controls and detected by anti-GAPDH and anti- $\beta$-actin primary antibodies (1:2000; Santa Cruz Biotechnology, Santa Cruz, CA, USA). The appropriate secondary antibodies were incubated with the nitrocellulose membrane for $2 \mathrm{~h}$ at room temperature. Chemiluminescent detection was performed to identify the immune bands (ECL Plus; Amersham Bioscience, Arlington Heights, IL, USA). Data were analyzed by densitometry using Quantity One 4.6.2 (Bio-Rad Laboratories, Berkeley, CA, USA).

\section{Immunofluorescence staining}

Immunofluorescence staining was performed on fixed frozen brain sections as previously described $[6,17]$. At $24 \mathrm{~h}$ after SAH induction, the mice were deeply anesthetized and transcardially perfused with PBS and 4\% PFA. The brains were rapidly isolated and postfixed in $4 \%$ PFA for $24 \mathrm{~h}$ and then soaked in $40 \%$ sucrose for 1 day. Coronal brain sections $(10 \mu \mathrm{m})$ were obtained using a cryostat (Leica CM3050S-3-1-1, Bannockburn, IL, USA) and permeabilized with $0.3 \%$ Triton X-100 in PBS for 30 min. Sections were blocked with 5\% donkey serum for $1 \mathrm{~h}$ and incubated at $4{ }^{\circ} \mathrm{C}$ overnight with anti-collagen IV (Abcam, Cambridge, UK) and Lectin antibodies (Vector Laboratories, Burlingame, CA) and then incubated with fluorescein isothiocyanate-conjugated secondary antibodies (Jackson Immunoresearch, West Grove, PA) and Dylight 649-conjugated streptavidin (Vector Laboratories, Burlingame, $\mathrm{CA}$ ) for $4 \mathrm{~h}$ at room temperature.

Brain capillaries were isolated as described above and then cytospun ( $500 \mathrm{~g}$ ) for $5 \mathrm{~min}$ onto Superfrost Plus precleaned glass microscopy slides. The microvessel fragments were then fixed using ICC Fixation Buffer (BD, Franklin Lakes, NJ, USA) for $15 \mathrm{~min}$ at room temperature. The microvessels were then rinsed with PBS and blocked in PBS containing 0.4\% Triton X-100 and 5\% Swine Serum (Vector Laboratories, Burlingame, CA, USA) for $1 \mathrm{~h}$ at room temperature followed by incubation with the following antibodies: anti-CypA (Abcam, Cambridge, UK), antiPDGFR $\beta$ (1:100, Abcam, Cambridge, United Kingdom), anti-CD13 (Novus Biologicals, Littleton, CO, USA), and anti-lectin (Vector Laboratories, Burlingame, CA, USA). The microvessels were subsequently incubated with fluorescein isothiocyanate-conjugated and Cy3-conjugated secondary antibodies (Jackson Immunoresearch, West Grove, PA, USA) as well as Dylight 649-conjugated streptavidin (Vector Laboratories, Burlingame, CA, USA) for $4 \mathrm{~h}$ at room temperature. The colocalization of CypA, the pericyte marker CD13 and Lectin was examined by a confocal laser scanning microscope (Zeiss, Oberkochen, Germany).

\section{Brain pericyte cultures and RNAi}

Pericyte culture was performed as previously described [6]. The brains were harvested from 30 mice (P1-P3) and digested in an enzymatic solution that contained 30 $\mathrm{U} / \mathrm{ml}$ papain and $40 \mu \mathrm{g} / \mathrm{ml}$ DNase I in HEPES balanced salt solution. Then, digestion was terminated in PBS containing 1.7 times the volume of $22 \%$ bovine serum albumin and centrifuged at $6000 \mathrm{~g}$ for $10 \mathrm{~min}$. Cells in the 
lower layer were resuspended in endothelial cell growth medium consisting of Hams F12 supplemented with 10\% FBS, heparin, ascorbic acid, L-glutamine, penicillin/ streptomycin, and endothelial cell growth supplement (ScienCell Research Laboratories, San Diego, CA, USA). Then, these cells were plated in a six-well plate coated with collagen I $(0.02 \%$, Sigma-Aldrich, St. Louis, MO, USA) for $2 \mathrm{~h}$ at $37^{\circ} \mathrm{C}$. The first passage was performed on the 7th-9th day. Subsequently, the cells were cultured in pericyte medium (ScienCell Research Laboratories, San Diego, CA, USA) containing 2\% FBS and could be used after the third passage. CypA siRNA, CD147 siRNA, or scrambled siRNA at a final concentration $100 \mathrm{nmol} / \mathrm{ml}$; Lipofectamine 2000 Transfection Reagent (Thermo Fisher Scientific, Waltham, MA, USA) at a final concentration 0.4 vol.\%; and Opti-MEM (Thermo Fisher Scientific, Waltham, MA, USA) at a final concentration 10 vol.\% were mixed with serum-free medium, according to the manufacturer's instructions. After incubation for $4 \mathrm{~h}$, the siRNA-containing medium was replaced with the standard pericyte culture medium, WB, zymography, Flow cytometry, ELISA was performed respectively at $24 \mathrm{~h}$ post-treatment. For identification via immunocytochemical analysis, the cells were treated under the same conditions as described above but then passaged onto collagen-coated slides and grown for 3 days unless otherwise indicated. Cultures were confirmed to be morphologically consistent with pericyte cultures, and they were also PDGFR $\beta$-positive, SMA-positive, CD13positive, GFAP-negative, AQP4-negative, TUJ1-negative, NeuN-negative, CD31-negative, Iba1-negative, and Olig2 -negative as previously reported $[6,9]$.

\section{Gelatinase zymography}

Gelatinase zymography was performed as previously described with modifications [18]. Briefly, isolated microvessels were homogenized in lysis buffer, and the pericytes conditioning media $(1.5 \mathrm{ml})$ was concentrated to 20-30 $\mu \mathrm{l}$ using microcon spin columns (Millipore, Bedford, MA). The samples were separated on an $8 \%$ polyacrylamide SDS gel containing $1 \mathrm{mg} / \mathrm{ml}$ gelatin. The gel was then soaked in zymogram renaturing buffer (Novex, Shanghai, China) for $30 \mathrm{~min}$ at room temperature and incubated for $16 \mathrm{~h}$ at $37{ }^{\circ} \mathrm{C}$ in zymogram developing buffer (Novex, Shanghai, China). To visualize the MMPs, the gel was stained with SimplyBlue Safe Stain (Invitrogen, Shanghai, China) for $30 \mathrm{~min}$. The gel was imaged using a CCD camera and analyzed using ImageJ software (NIH, Bethesda, MD, USA).

\section{Flow cytometry}

The cells were incubated with the blank control, vehicle (DMSO), Amiloride $(100 \mu \mathrm{M})$, Dynasore $(80 \mu \mathrm{M})$, Genistein $(200 \mu \mathrm{M})$, Chloroquine $(200 \mu \mathrm{M})$, and Chlorpromazine
$(30 \mu \mathrm{M})$ for $1 \mathrm{~h}$, and scrambled siRNA or CD147 siRNA was transfected for $48 \mathrm{~h}$. The cells were then washed and incubated with CypA-FITC $(1 \mu \mathrm{g} / \mathrm{ml}$, Novoprotein, Shanghai, China) for $1 \mathrm{~h}$ except for the blank group. The cells were harvested in aliquots of $1 \times 10^{6}$ cells per $100 \mu \mathrm{l}$. The positive cell count and FITC-positive cells percentage were determined via flow cytometry (BD, Franklin Lakes, NJ, USA). The data were processed using FlowJo 7.6.1 (BD, Franklin Lakes, NJ, USA).

\section{ELISA}

The levels of CypA and MMP9 in the supernatant of the cultured pericyte samples were measured by ELISA according to the manufacturer's protocol for CypA (CusaBio, Wuhan, China) and MMP9 (R\&D system, Minneapolis, MN, USA) detection. The cells were treated with either a vehicle (sterile saline), $\mathrm{Hb}(10 \mu \mathrm{M}), \mathrm{Hb}+$ vehicle, $\mathrm{Hb}+$ CypA, $\mathrm{Hb}+$ scrambled siRNA, $\mathrm{Hb}+$ CypA siRNA, $\mathrm{Hb}+$ CypA + scrambled siRNA, Hb + CypA + CD147 siRNA, $\mathrm{Hb}+$ CypA + vehicle (DMSO), or Hb + CypA + CPZ (30 $\mathrm{Mm})$. For the measurement of MMP9, the cells were pretreated for $48 \mathrm{~h}$ with scrambled siRNA, CypA siRNA, or CD147 siRNA, and the other inhibitors were added $24 \mathrm{~h}$ before collecting the cell supernatants. A total of $1 \times 10^{6}$ cells were dispensed in triplicate onto 12 -well plates and cultured for different times according to the manufacturer's recommendations.

\section{Statistical analysis}

Data are shown as the mean $\pm \mathrm{SD}$. Chi-square tests were used for the behavior score analyses. A one-way analysis of variance (ANOVA) followed by Tukey's multiple comparisons test was used to compare the different groups. SPSS 18 was used to analyze the data, and $P<0.05$ was considered significant.

\section{Results \\ CypA expression in pericytes after SAH}

To investigate the expression of CypA in the pericytes after $\mathrm{SAH}$ induction, we detected the protein levels of CypA in the microvessels of the brain specimen from the mice after $\mathrm{SAH}$ induction and in the cultured pericytes after $\mathrm{Hb}$ stimulation. The Western blot analysis illustrated a significant elevation of CypA in the ipsilateral/left hemisphere 12 to $72 \mathrm{~h}$ after SAH (Fig. 1a). Furthermore, immunohistochemical staining at $24 \mathrm{~h}$ (the peak of CypA expression) after SAH showed that CypA expression was colocalized with the pericyte markers, Lectin and PDGFR $\beta / C D 13$, in the left/ipsilateral cortex (Fig. 1b). The Western blot analysis of the cultured pericytes also demonstrated a significant elevation of intracellular CypA after $\mathrm{Hb}$ stimulation at all time points up to $72 \mathrm{~h}$ except at $3 \mathrm{~h}$ (Fig. 1c), and confirmed by immunohistochemical staining at $24 \mathrm{~h}$ after $\mathrm{Hb}$ stimulation (Fig. 1d). 


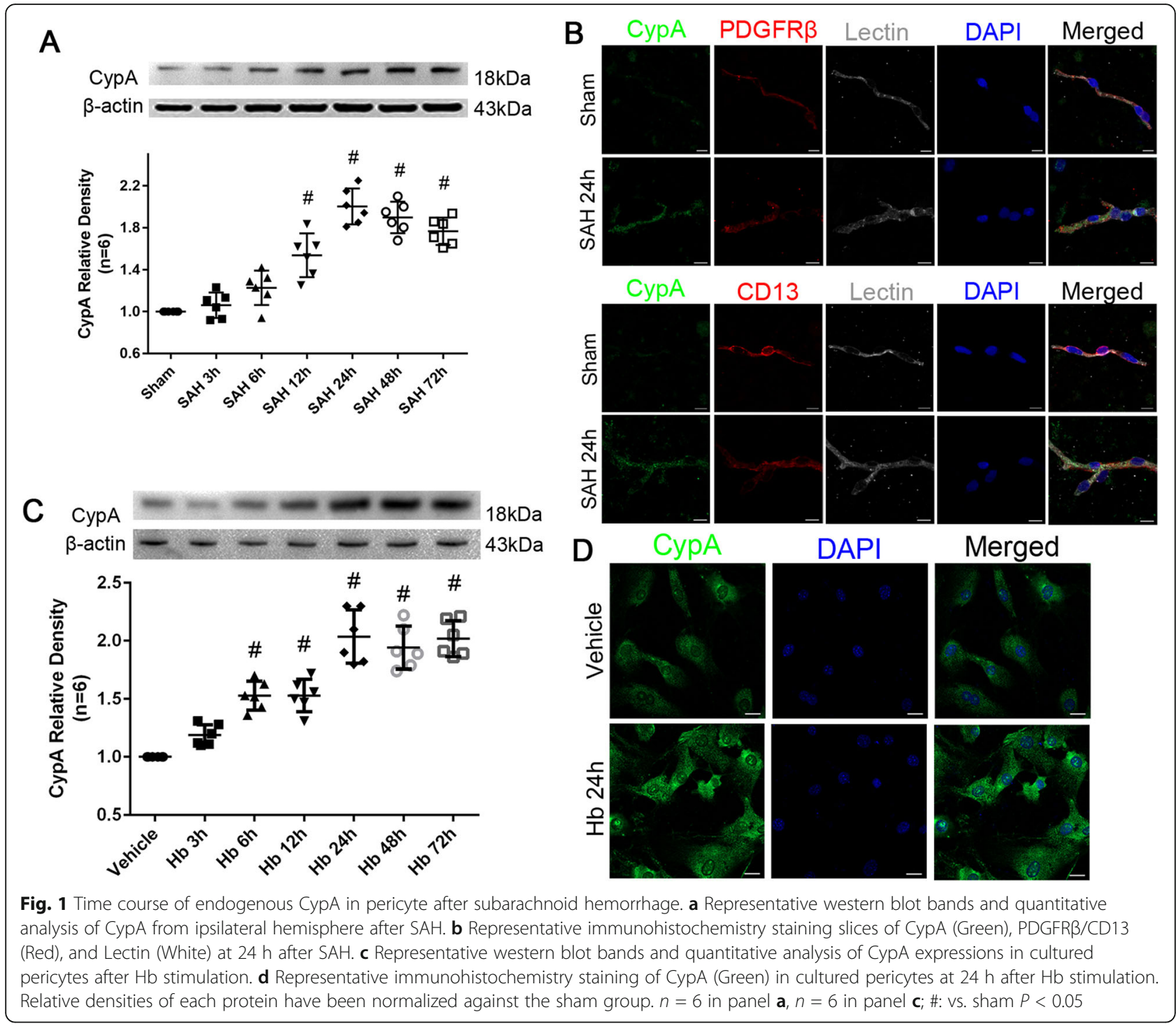

Neurological deficit changes and blood-brain barrier disruption after $\mathrm{SAH}$ in $\mathrm{CypA}^{-1-}$ mice

To confirm the pivotal role of CypA, we utilized CypA knockout $\left(\mathrm{CypA}^{-/-}\right)$mice in the present study. There was no statistical difference of $\mathrm{SAH}$ grade between wildtype and $\mathrm{CypA}^{-/-}$mice in sham and SAH groups (Fig. 2a), which indicated the SAH models were consistent and comparable. The SAH mouse model exhibited significant neurological deficits at $24 \mathrm{~h}$ after SAH (Fig. 2a, b). However, compared with the flox/flox SAH mice, the CypA ${ }^{-/-}$ mice exhibited a better performance in the modified Garcia test (Fig. 2a) and the beam balance test (Fig. 2b). Further investigation indicated that the Evans blue and cadaverine extravasation in the $\mathrm{CypA}^{-/-}$mice was less than that in the $\mathrm{CypA}^{+/+}$(flox/flox) mice $24 \mathrm{~h}$ after SAH (Fig. 2c, e, f), which was verified by the fluorescence imaging of the Evans blue extravasation at the same timepoint after SAH (Fig. 2d). Immunohistochemical staining showed that the continuous endothelial cell layer (Lectin) and basement membrane (Collagen IV) were disrupted in wildtype $\mathrm{SAH}$ mice at $24 \mathrm{~h}$ after $\mathrm{SAH}$, which was alleviated in $\mathrm{CypA}^{-1-} \mathrm{SAH}$ mice (Fig. 2e).

\section{Effects of exogenous recombinant CypA and specific} inhibition of CypA expression after SAH

The CypA siRNA used in the present study could effectively inhibit the CypA expression after SAH (Fig. 3a, b). And comparisons of the SAH grading score did not reveal significant differences among the groups either at 24 or $72 \mathrm{~h}$ after SAH. Compared with the Sham group, all SAH mouse models showed significant neurological impairment as assessed by the modified Garcia test both at 24 and $72 \mathrm{~h}$ after SAH induction (Fig. 3c-f). Further analysis indicated that the $\mathrm{SAH}+\mathrm{CypA}$ mice had more severe neurological deficits at 24 and $72 \mathrm{~h}$ after $\mathrm{SAH}$ compared with the $\mathrm{SAH}+$ vehicle mice (Fig. 3c-f). 


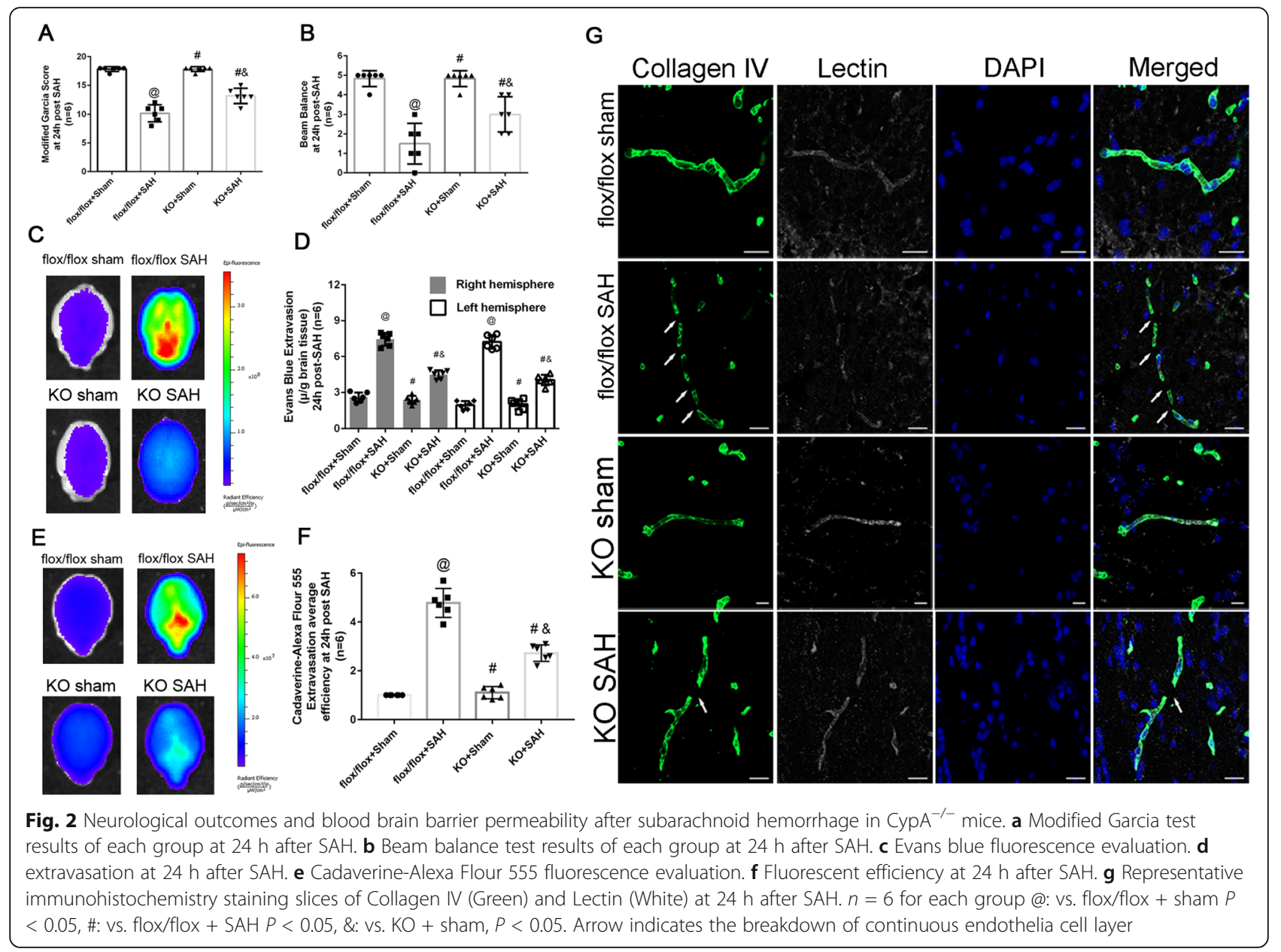

However, the CypA siRNA treatment showed greater improvements in neurological deficits compared with the $\mathrm{SAH}+$ vehicle, $\mathrm{SAH}+\mathrm{CypA}$, and $\mathrm{SAH}+$ scrambled siRNA groups at both 24 and $72 \mathrm{~h}$ after SAH (Fig. 3c-f). For the beam balance test, all SAH mouse models showed significant neurobehavioral dysfunction compared with the Sham group at 24 and $72 \mathrm{~h}$ after SAH except the SAH + CypA siRNA group at $72 \mathrm{~h}$ after SAH (Fig. 3c-f). Similarly, for the modified Garcia test, mice from the SAH + CypA group exhibited significantly worse neurobehavioral functions compared with the mice from the SAH + vehicle groups (Fig. 3c-f). In addition, the CypA siRNA treatment significantly alleviated neurological impairments at 24 and $72 \mathrm{~h}$ after SAH (Fig. 3c-f).

Mice from the SAH + vehicle, SAH + CypA, and SAH + scrambled siRNA groups showed increased brain water content in both hemispheres $24 \mathrm{~h}$ after SAH compared with the mice from the Sham group (Fig. 3g). The CypA treatment did not increase the brain water content compared with the SAH + vehicle group, whereas the CypA siRNA pretreatment significantly reduced the brain water content in both hemispheres at $24 \mathrm{~h}$ after SAH (Fig. 3g).
Furthermore, the SAH mouse models showed more Evans blue extravasation than the mice in the Sham group in both hemispheres (Fig. 3h). The SAH + CypA mice exhibited increased Evans blue leakage compared with the $\mathrm{SAH}+$ vehicle mice, whereas the CypA siRNA pretreatment significantly reduced the Evans blue extravasation compared with the $\mathrm{SAH}+$ scrambled siRNA group (Fig. $3 \mathrm{~h}$ ). In the immunohistochemical staining images, continuous Lectinpositive endothelial cells and a collagen IV-positive basement membrane were observed in the Sham mice. However, in the SAH + vehicle, SAH + CypA, and SAH + scrambled siRNA groups, the Lectin and collagen IV structures were disrupted at $24 \mathrm{~h}$ after SAH, and the CypA siRNA pretreatment effectively reduced these damages (Fig. 3i).

The expression of MMP9 was significantly increased $24 \mathrm{~h}$ after SAH and decreased after the CypA siRNA pretreatment (Fig. 4a, b). Gelatin zymography showed that the MMP9 levels were elevated in the SAH + vehicle, SAH + CypA, and SAH + scrambled siRNA groups compared with the Sham group, and these levels were significantly elevated in the SAH + CypA group compared with the SAH + vehicle and $\mathrm{SAH}+$ scrambled siRNA groups (Fig. 4e, f). 


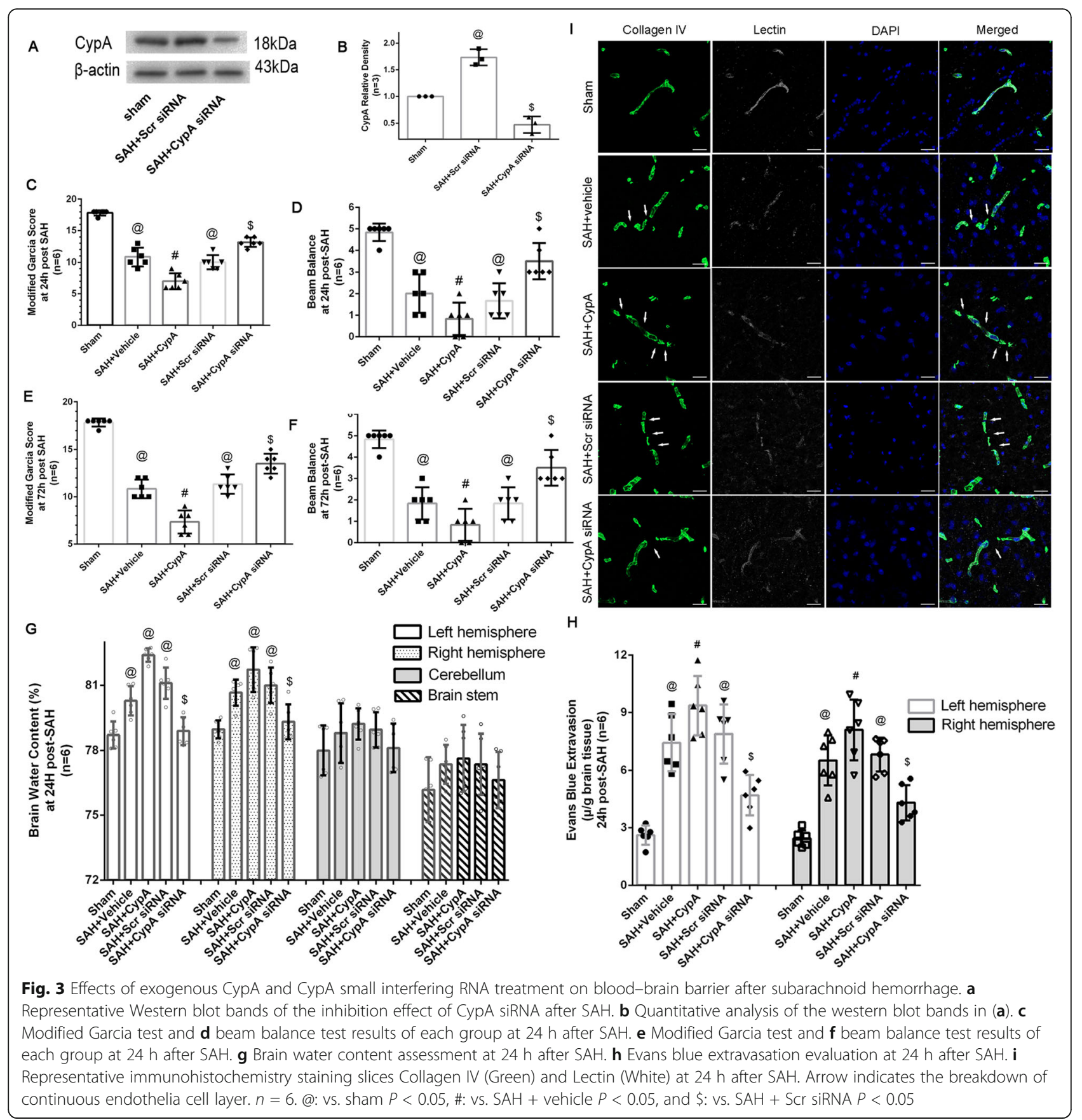

However, the CypA siRNA pretreatment decreased the MMP9 levels at $24 \mathrm{~h}$ after SAH (Fig. 4a, b). Furthermore, the expression of P-p65 was significantly increased $24 \mathrm{~h}$ after SAH, whereas the ZO-1, Collagen IV, Occludin, and Claudin 5 levels were significantly reduced at $24 \mathrm{~h}$ after SAH (Fig. 4a, c, g-k). While total p65 did not show significant differences among groups. The CypA siRNA pretreatment decreased the expression levels of P-p65 and preserved the ZO-1, Collagen IV, Occludin, and Claudin 5 levels compared with the $\mathrm{SAH}+$ vehicle group (Fig. 4a, c, g-k).

\section{CypA secretion and endocytosis in cultured pericytes}

To determine the role of CypA in the pericyte- and MMP9-associated blood--brain barrier disruption, we cultured primary pericytes and evaluated the intracellular and extracellular CypA expression. Intracellular CypA expression in the cultured pericytes was significantly increased at 6 to $72 \mathrm{~h}$ after $\mathrm{Hb}$ stimulation, with the peak expression occurring at 24 to $72 \mathrm{~h}$ after stimulation (Fig. 1c), which was confirmed by the immunohistochemical staining of CypA in the cultured pericytes at $24 \mathrm{~h}$ after $\mathrm{Hb}$ stimulation (Fig. 1d). In addition, ELISA 


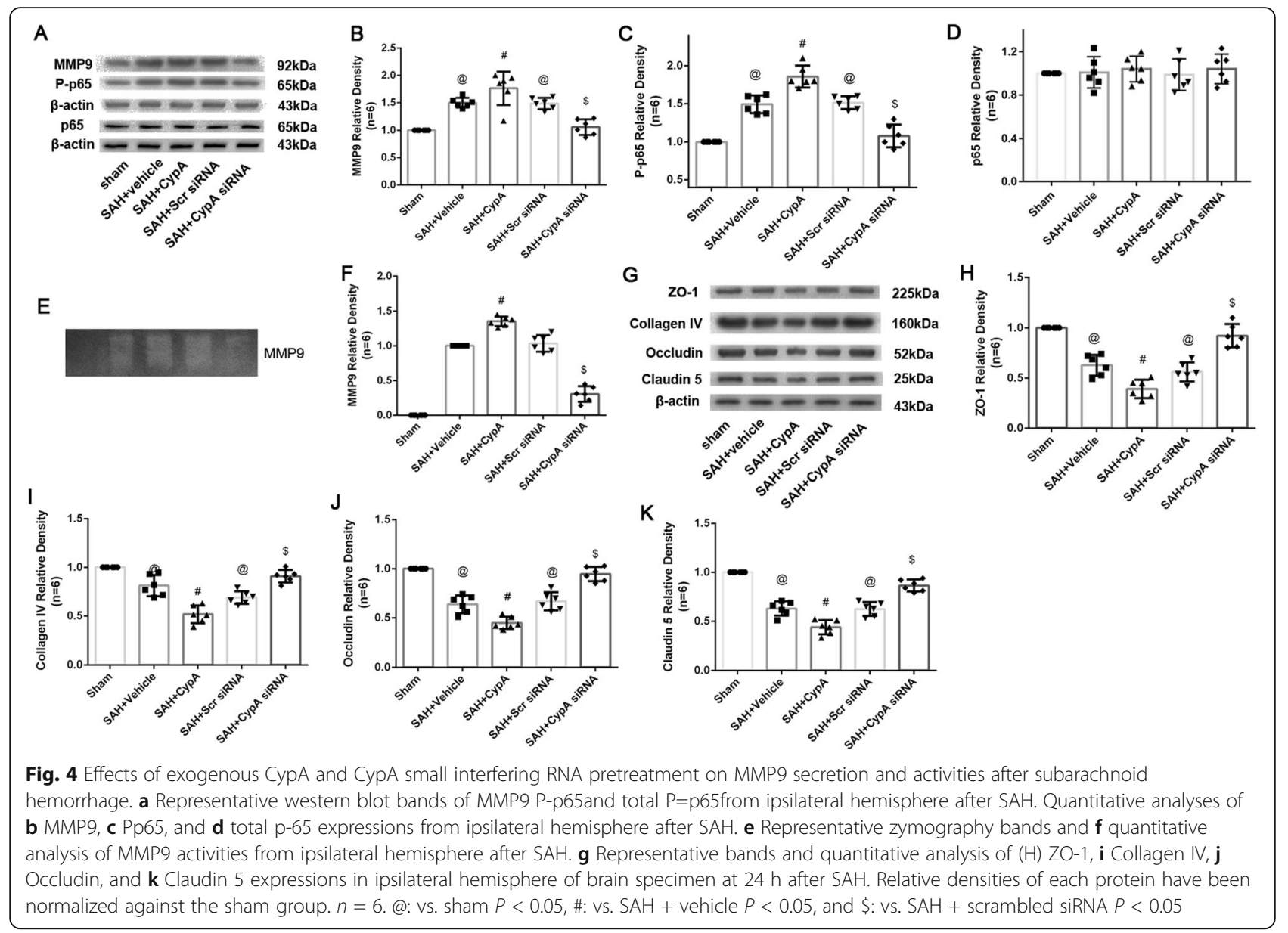

data indicated secreted CypA in the culture media was significantly increased at 6 to $72 \mathrm{~h}$ after $\mathrm{Hb}$ stimulation, and peak at 24 to $48 \mathrm{~h}$ (Fig. 5a). The CypA concentrations in the culture media were reduced at $24 \mathrm{~h}$ after $\mathrm{Hb}$ stimulation in the CypA siRNA-pretreated group compared with the scrambled siRNA-pretreated group (Fig. 5b).

In the present study, exogenous CypA was conjugated with FITC to investigate the endocytosis of CypA in the cultured pericytes. Confocal microscopy observations indicated that CypA-FITC was endocytosed into the cultured pericytes $1 \mathrm{~h}$ after CypA-FITC incubation and continued for up to $3 \mathrm{~h}$ (Fig. 5c), indicating that cypa could be endocytosed by pericyte physiologically. Further flow cytometry analyses indicated that this endocytosis could be inhibited by chlorpromazine but not by amiloride, dynasore, genistein, or chloroquine (Fig. 5d).

Secretion inhibitor Exo1 and Exo2 was used to study process of CypA secretion from pericyte. We found both Exo1 and Exo2 reducing CypA in medium after $\mathrm{Hb}$ stimulation (Fig. 5e).

Furthermore, the MMP9 concentrations and activities in culture media were significantly increased after $\mathrm{Hb}$ simulation versus vehicle group, and even much higher in $\mathrm{Hb}+\mathrm{CypA}$ group versus solo $\mathrm{Hb}$ stimulation group. By pretreated with CypA siRNA, the MMP9 concentrations and activities in culture media were significantly lower than $\mathrm{Hb}$ group and $\mathrm{Hb}+\mathrm{CypA}$ group (Fig. $5 \mathrm{f}-\mathrm{h}$ ). Both Exo1 and Exo2 treatment significantly lowered MMP9 concentration in cultured medium post $\mathrm{Hb}$ stimulation (Fig. 5i).

\section{CD147 expressions in pericytes after SAH}

CD147 has been reported to serve as the receptor for CypA; therefore, we also evaluated CD147 expression in the pericytes after SAH induction. We found that CD147 was significantly increased in cerebral microvessels after SAH except at $6 \mathrm{~h}$, and a peak was observed at $24 \mathrm{~h}$, and it was sustained until $72 \mathrm{~h}$ after SAH as confirmed by immunohistochemical staining of Lectin and PDGFR $\beta / C D 13$ at $24 \mathrm{~h}$ after SAH (Fig. 6a, b). Similarly, the cultured pericytes also overexpressed CD147 from 12 to $72 \mathrm{~h}$ after $\mathrm{Hb}$ stimulation, with a peak observed at $24 \mathrm{~h}$, and the expression pattern was also confirmed by immunohistochemical staining with a CD147 antibody (Fig. 6c, d). 


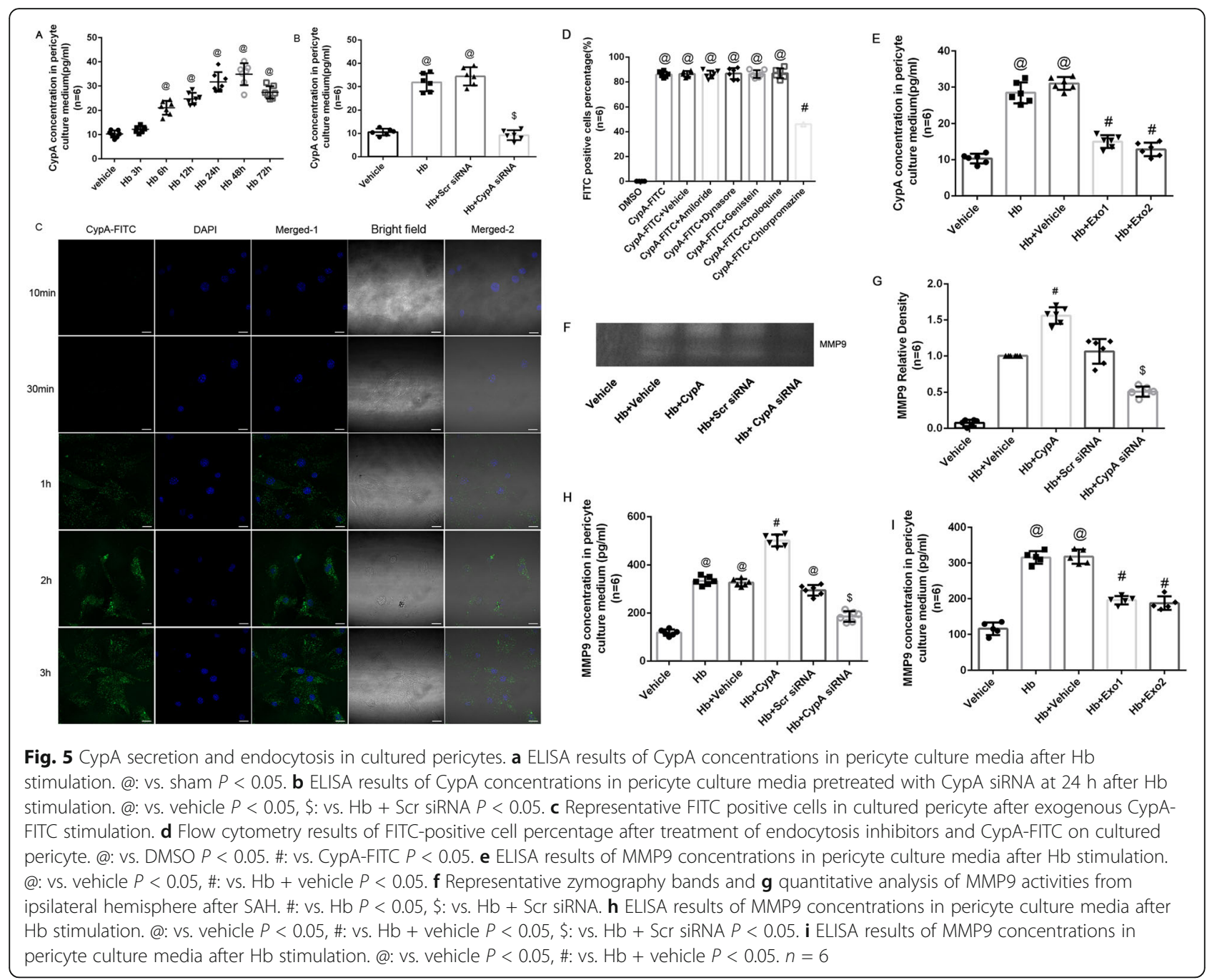

\section{CD147 inhibition reduced MMP9 secretion without affecting CypA endocytosis}

Significant differences in the FITC-positive cells percentage of CypA-FITC were not observed in the CD147 siRNA-pretreated cultured pericytes at $1 \mathrm{~h}$ after incubation (Fig. 7a). However, the MMP9 concentration in the culture media was significantly increased at $24 \mathrm{~h}$ after $\mathrm{Hb}+$ CypA incubation and reduced by the CD147 siRNA pretreatment (Fig. 7b). In addition, chlorpromazine had an inhibitory effect on CypA endocytosis, but it did not reduce MMP9 concentrations in the supernatant of the culture media compared with the $\mathrm{Hb}+\mathrm{CypA}+$ vehicle group (Fig. 7b). Gelatin zymography of the media supernatants also showed that $\mathrm{Hb}+\mathrm{CypA}$ incubation increased the pro-MMP9 activity compared with the $\mathrm{Hb}$ + vehicle group, whereas the $\mathrm{Hb}+$ CypA siRNA group showed less pro-MMP9 activity than the $\mathrm{Hb}+$ scrambled siRNA group. CD147 siRNA significantly reduced the pro-MMP9 activity following $\mathrm{Hb}+\mathrm{CypA}$ incubation compared with the $\mathrm{Hb}+\mathrm{CypA}+\mathrm{CD} 147$ siRNA group, whereas significant differences were not observed between the $\mathrm{Hb}+\mathrm{CypA}+$ vehicle and $\mathrm{Hb}+\mathrm{CypA}+$ chlorpromazine groups (Fig. $7 \mathrm{c}, \mathrm{d}$ ).

\section{Effects of exogenous recombinant CypA and specific inhibition of CD147 expression after SAH}

The CD147 siRNA used in the present study could effectively inhibit the CD147 expression after SAH (Fig. 8a, b), and comparisons of the $\mathrm{SAH}$ grading score did not reveal significant differences among the groups at $24 \mathrm{~h}$ after SAH. Mice from the SAH + CypA + CD147 scrambled siRNA and $\mathrm{SAH}+\mathrm{CypA}+\mathrm{CD} 147$ siRNA groups showed significant neurological impairment during the modified Garcia test and the beam balance test at $24 \mathrm{~h}$ after SAH compared with the Sham group (Fig. 8c, d). However, the SAH + CypA + CD147 siRNA group showed alleviated neurological impairment compared with the SAH + CypA + CD147 scrambled siRNA groups (Fig. 8c, d). In addition, the $\mathrm{SAH}+\mathrm{CypA}+\mathrm{CD} 147$ scrambled siRNA group showed increased brain water content in both hemispheres 


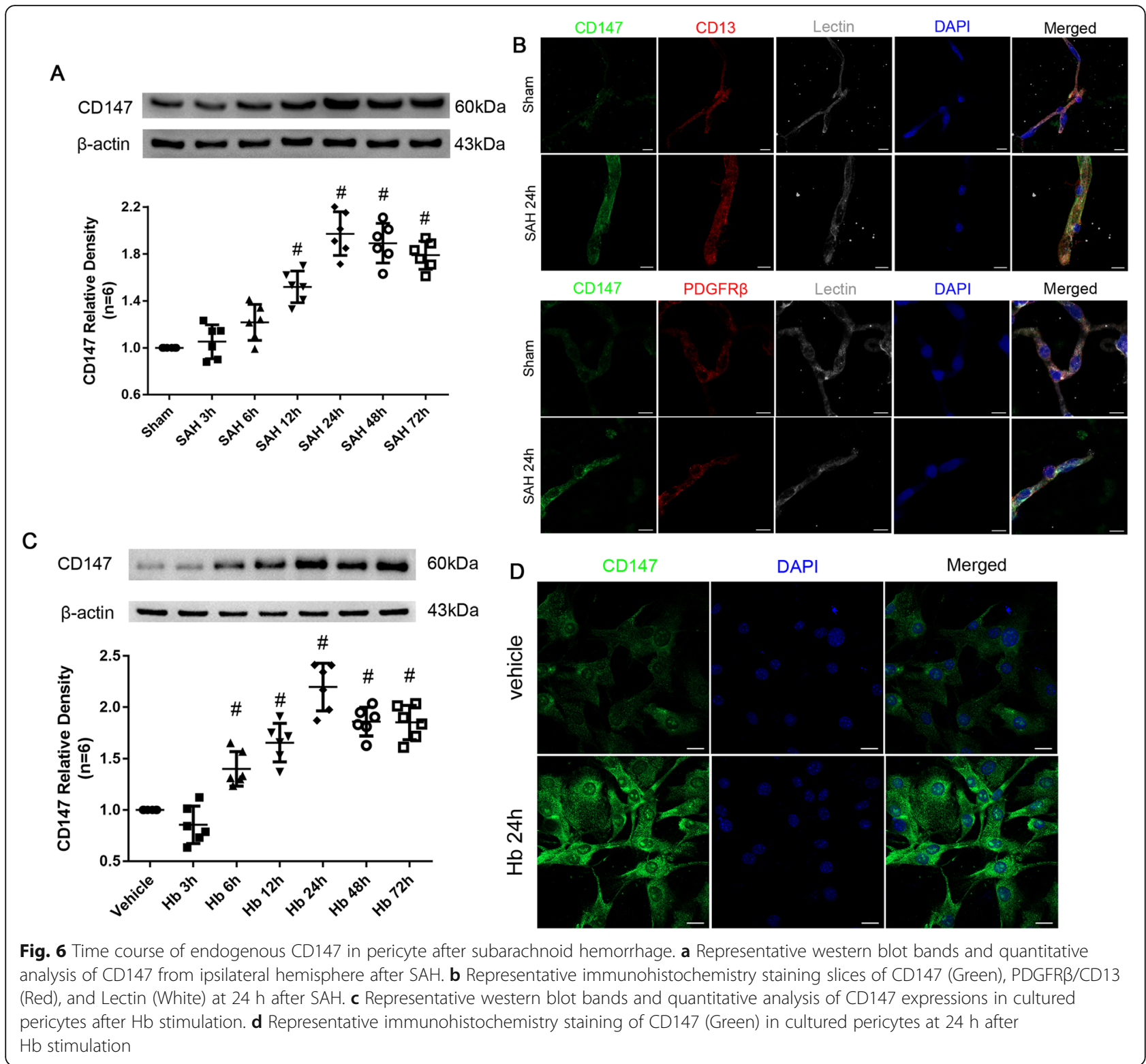

at $24 \mathrm{~h}$ after SAH (Fig. 8e). The SAH + CypA + CD147 siRNA pretreatment significantly reduced the brain water content in both hemispheres at $24 \mathrm{~h}$ after SAH (Fig. 8e). Furthermore, the SAH + CypA + CD147 siRNA group showed less Evans blue extravasation than the $\mathrm{SAH}+\mathrm{CypA}$ + CD147 scrambled siRNA group in both hemispheres at $24 \mathrm{~h}$ after SAH, although the Evans blue extravasation level was still higher than that in the Sham group (Fig. 8f).

Immunohistochemical staining showed that the continuous endothelial cell layer (Lectin) and basement membrane (collagen IV) were disrupted in the SAH + CypA + CD147 siRNA scramble group at $24 \mathrm{~h}$ after $\mathrm{SAH}$, which was similar to the phenomenon observed in the SAH + vehicle and SAH + CypA groups; however, the CD147 siRNA pretreatment effectively reduced those damages (Fig. 8g). Further analysis indicated that the expression of P-p65 and MMP9 was significantly increased and total p65 was not altered at $24 \mathrm{~h}$ after SAH (Fig. 9a-d). In addition, gelatin zymography confirmed that the tight junctions and basement membranes were disrupted by MMP9 activity after SAH but alleviated by the CD147 siRNA pretreatment compared with the SAH + CypA + CD147 scrambled siRNA group (Fig. 9e, f). And the ZO-1, collagen IV, Occludin, and Claudin 5 was significantly reduced at $24 \mathrm{~h}$ after SAH (Fig. 9g-k). The CD147 siRNA pretreatment decreased the expression levels of P-p65 and MMP9 and preserved the expression levels of ZO-1, Collagen IV, Occludin, and Claudin 5 compared with that of the SAH + CypA + CD147 siRNA group (Fig. 9g-k). 


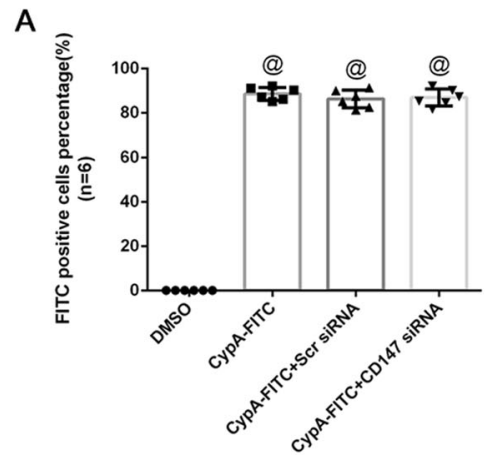

B
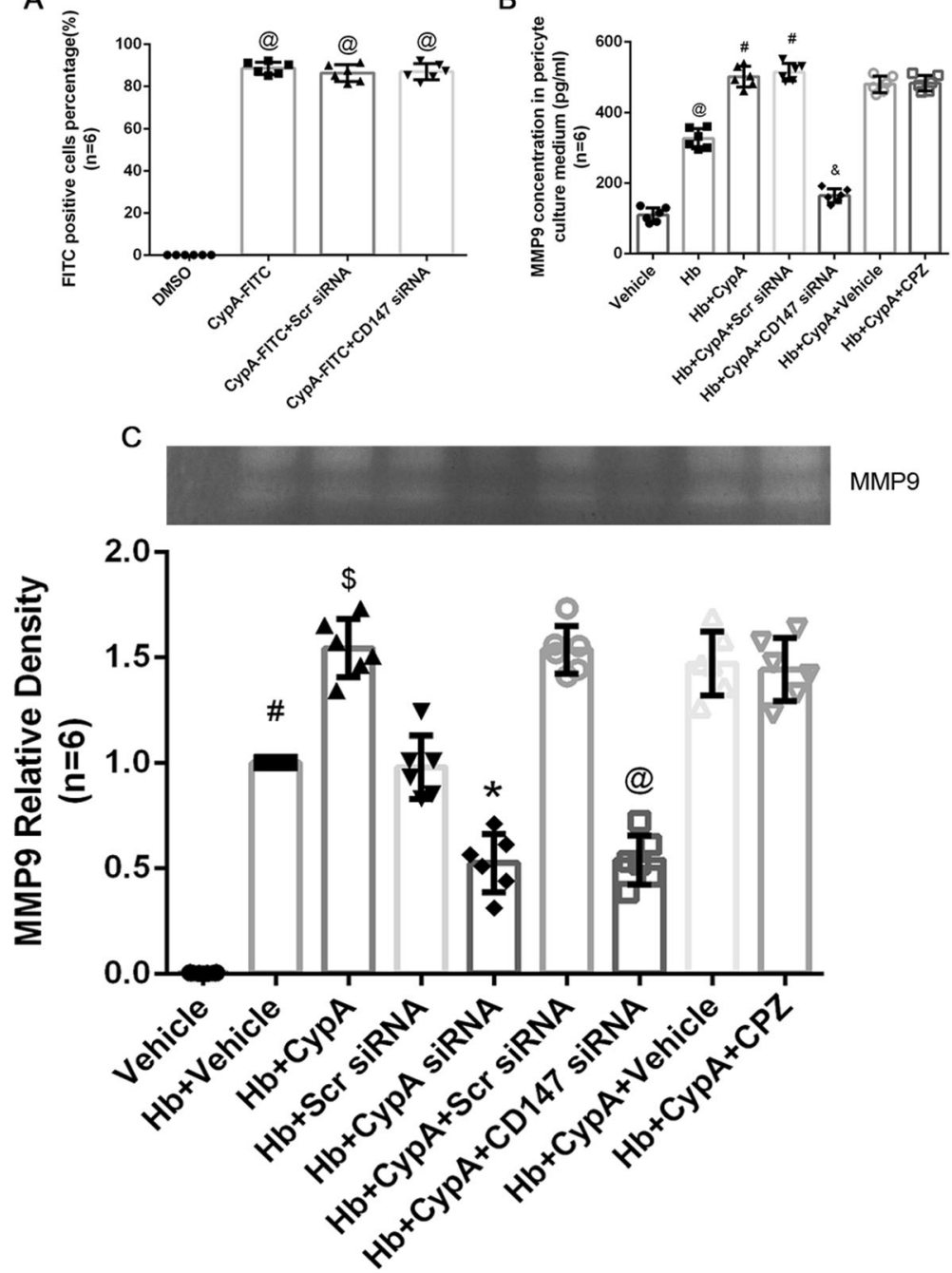

Fig. 7 CD147 inhibition reduced MMP9 secretion without effecting CypA endocytosis. a Representative FITC-positive cell percentage after treatment of CD147 siRNA and CypA-FITC on cultured pericyte. $n=6$. @: vs. DMSO $P<0.05$. b ELISA results of MMP9 concentrations in pericyte culture media after exogenous CypA, CD147 siRNA, and chlorpromazine (CPZ) treatment. $n=6$. @: vs. Hb $P<0.05$. \#: vs. Hb + CypA $P<0.05$, \&: vs. Hb + CypA + Scr siRNA $P<0.05$. \$: vs. $\mathrm{Hb}+\mathrm{CypA}+$ vehicle $P<0.05$. c Representative zymography bands and quantitative analysis of MMP9 of cultured pericyte supernatant after exogenous CypA, CD147 siRNA, and CPZ treatment. Relative densities of each group have been normalized against the first group. $n=6$. \#: vs. Hb + vehicle $P<0.05$. \$: vs. Hb + CypA $P<0.05$, *: vs. Hb + Scr siRNA $P<0.05$, @: vs. Hb + CypA siRNA $P<0.05$

\section{Discussion}

The present study demonstrated that both intracellular CypA and CypA secretion increased after $\mathrm{SAH}$ and could activate its receptor CD147 and the downstream NF- $\mathrm{kB}$ inflammatory pathway to induce MMP9 expression and proteolytic functions for the degradation of endothelium tight junction proteins and basal membranes. CypA served as an autocrine or a paracrine ligand for its receptor, CD147 (Fig. 10). Although CypA could be endocytosed by pericytes, the specific endocytosis inhibitor chlorpromazine did not have an effect on MMP9 activation following blood-brain barrier disruption. However, the specific knockdown of CD147 could reverse the harmful effects of CypA expression in the pericytes on the blood-brain barrier integrity after $\mathrm{SAH}$.
The blood-brain barrier is a highly selective neurovascular structure that can prevent harmful substances from entering the brain [19]. After SAH and other acute central nervous system injuries, increased blood-brain barrier permeability could lead to worse secondary pathophysiological changes by exposing neurons and other support cells in the vascular neural network to blood metabolites, and it could also lead to vasogenic brain edema, which causes further deterioration [20,21]. Based on the intimate connections between pericytes and endothelial cells, the pericytes have been shown to play a substantial role in the development of blood-brain barrier tight junctions and paracellular permeability [22]. Pericytes can form intercellular blood-brain barrier tight 


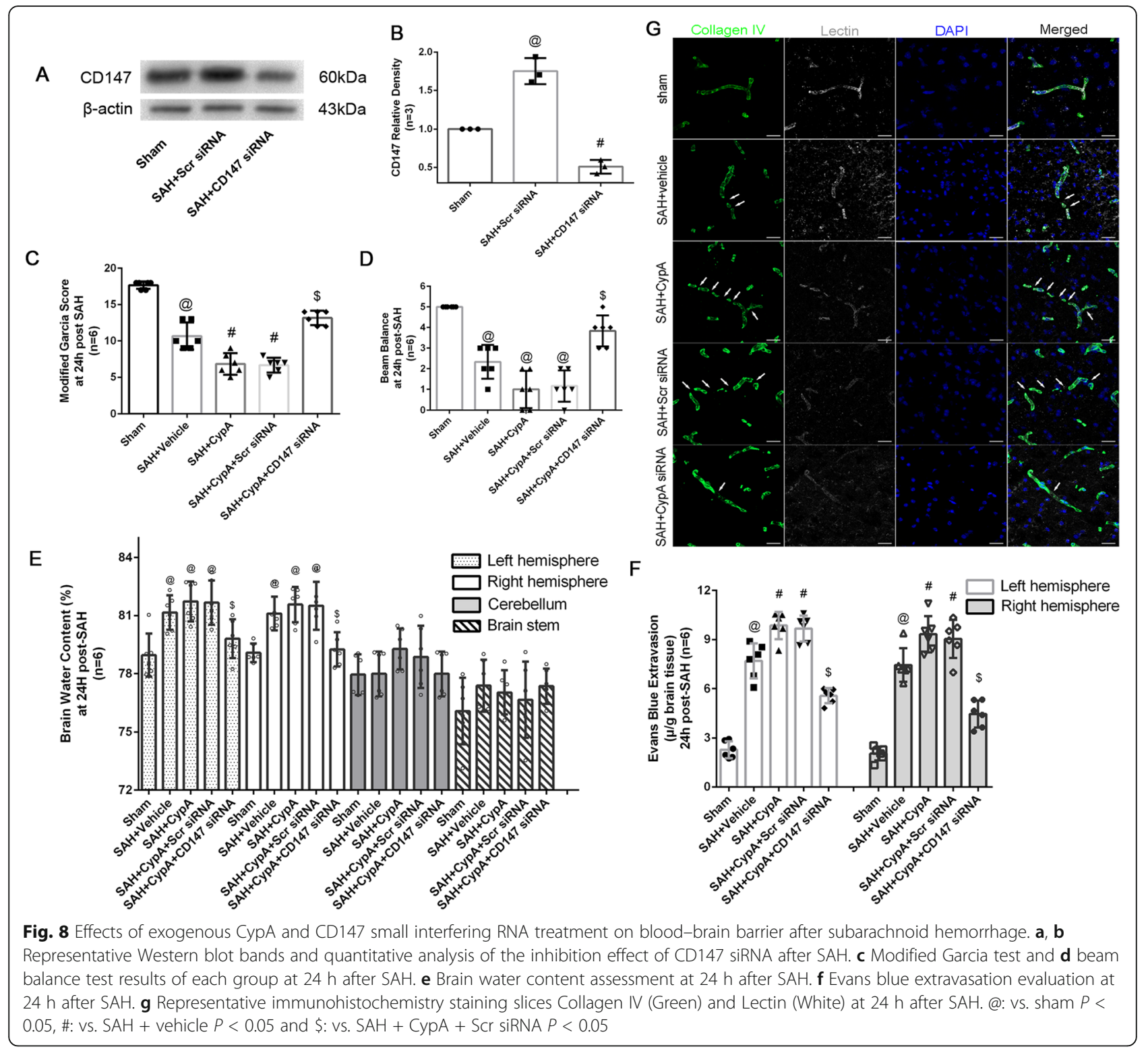

junctions [23]. In addition, pericytes also contribute to basal lamina formation by synthesizing collagen type IV, glycosaminoglycans, and laminin [24]. An inability to recruit pericytes to the endothelium in PDGF-B knockout mice results in severe vascular abnormalities, plasma leakage, and microaneurysm formation, indicating that pericytes maintain blood-brain barrier functions [3, 25]. However, the response of normally developed pericytes in the adult brain to the pathophysiological changes after $\mathrm{SAH}$ is not well understood. Because of the well-known pearl-like contraction in SAH patients with vasospasm, previous researchers as well as our group have studied the contractile functions of pericytes and their role in regulating microcirculation after $\mathrm{SAH}[6]$. In the present study, our data demonstrated the harmful effects of pericytes on the blood-brain barrier integrity after SAH. Cultured pericytes have recently been shown to produce matrix metalloproteinases after thrombin stimulation, and matrix metalloproteinases are key mediators of blood-brain barrier disruptions after SAH [26]. In addition, a recent study suggested that pericytes may be harmful for neurovascular integrity, which results in neuronal degeneration in apolipoprotein E-deficient mice [7]. Additional direct evidence was obtained with a two-photon microscope, and the results indicated that pericytes contribute to rapid and localized proteolytic degradation around pericyte somata during ischemia [8].

Cyclophilins are a protein family of highly conserved and ubiquitous proteins with peptidylprolyl isomerase activity [27]. The most distributed cyclophilin in humans 

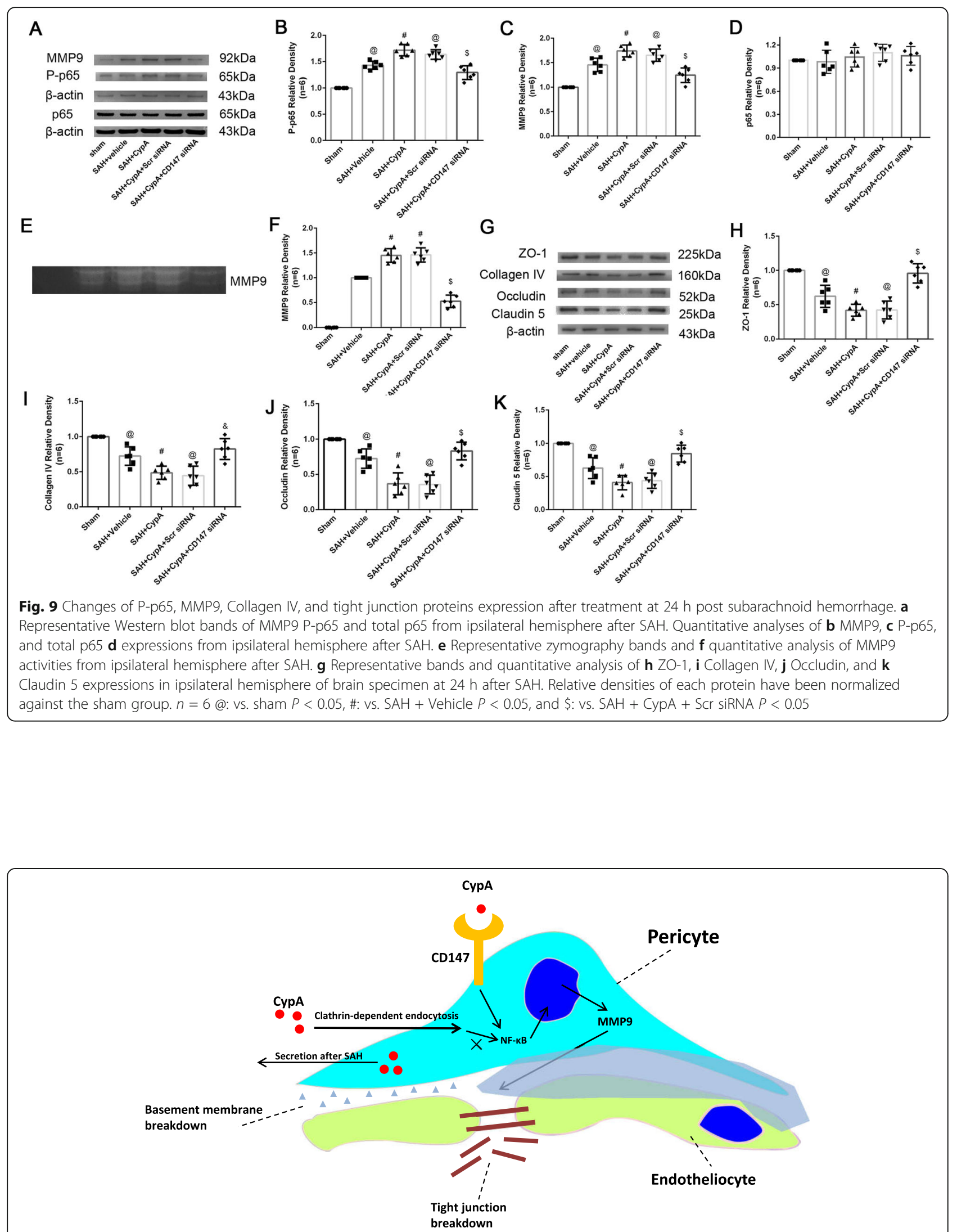

Fig. 10 CypA signal in pericytes induce rapid BBB disruption via CD147/NF-KB/MMP9 pathway after SAH 
is CypA, which is primarily expressed intracellularly and could be secreted from cells in response to various inflammatory stimulations after hypoxia, infection, and oxidative stress $[27,28]$. Previous studies have demonstrated that CypA activity is a modulator of $\mathrm{CD}^{+} \mathrm{T}$ cell signal transduction following cytokine production [29]. However, the exact mechanism underlying CypA secretion from the intracellular to extracellular space has not been well described. Previous studies have shown that CypA can be secreted from vascular smooth muscle cells via Rho guanine triphosphatease-myosin II activation/ actin remodeling-regulated vesicle transport [30, 31]. Another study has shown that the acetylation of CypA is required for its secretion into the extracellular space in vascular smooth muscle cells in response to oxidative stress [32]. And neuron was also found to express CypA [33]. Because reactive oxygen species could be generated in the central nervous system after SAH [34], we hypothesized that CypA may be secreted from pericytes and other cells in response to oxidative stress post-SAH. Exo 1-inhibited vesicular traffic from the endoplasmic reticulum to the Golgi might by interfere with GEFstimulated GDP/GTP exchange on ARF [35]; Exo2 disrupts the Golgi apparatus and interferes the secretory cargo exit from the endoplasmic reticulum [36]. Our data showed both Exo1 and Exo2 could efficiently reduce CypA secretion from pericyte, but whether these effects were identical to reported mechanism is unknown; further study is needed to illustrate to detail mechanism.

Our results indicated that extracellular CypA can be endocytosed by pericytes, which is a finding that has rarely been reported. M Carpentier et al. once illustrated that cyclophilin B could bind to two types of binding sites at the surface of capillary endothelial cells and showed that one type is involved in the endocytosis process [37]. Sanglifehrin A, a CypA-binding immunosuppressive drug, could reduce Lectin-mediated endocytosis in dendritic cells, which may occur via the decreased expression of C-type Lectins [38, 39]. A subsequent study demonstrated that the endocytosis gene END3 plays a pivotal role in the internalization of extracellular proteins/vesicles in response to glucose, which could rapidly decrease the extracellular CypA level under proper glucose conditions, which presumably occurs by enhancing CypA endocytosis [40]. However, additional evidence on the regulation of CypA endocytosis is required. The present study sought to investigate the role of CypA signaling in pericyte-associated bloodbrain barrier disruption after $\mathrm{SAH}$, and our data demonstrated that chlorpromazine (chlorpromazine: an inhibitor for clathrin-dependent endocytosis, but not by amiloride: macropiocytosis, dynasore: an inhibitor for dynamin-dependent endocytosis, genistein: an inhibitor for caveolin-dependent endocytosis, or chloroquine:an inhibitor for low pH-dependent endocytosis) could effectively inhibit CypA endocytosis by pericytes and showed that this process was not associated with the MMP9-mediated blood-brain barrier disruption after SAH. We focus on whether extracellular CypA induce MMP9 expression but not which specific progress of endocytosis CypA enter into pericyte.

However, the well-known receptor CD147, which is also known as the extracellular matrix metalloproteinase inducer (EMMPRIN), mediated this pathophysiological process [41]. CD147 is expressed in various types of cells and can crosstalk with integrin, annexin, caveolin, osteopontin, cyclophilin, etc. [41-45]. However, the functions of CD147 require further clarification. Previous studies have indicated that CD147 is expressed on the surface of normal endothelial cells in the central nervous system and showed that its expression could be induced in Alzheimer's disease, in brain tumors, and following ischemic brain injury [41, 42, 45, 46]. Because CD147 was demonstrated to induce MMP-1 expression in co-cultured tumor cells and fibroblast cells, it was considered an MMP inducer $[41,45]$. Further analysis showed that CD147 colocalized with MMP9 protein and displayed activity in experimental autoimmune encephalomyelitis brain tissues. After treatment with CD147-blocking antibodies, the improved outcome in the experimental autoimmune encephalomyelitis mice was associated with diminished MMP9 proteolytic activity [42]. More recently, CypA-CD147 signaling was demonstrated to be neuroprotective in both in vitro oxidative and ischemic neuron injury as well as in vivo early brain injury after SAH [33, 47]. S Chen et al. showed that MMPs are also involved in CypA/CD147-induced blood-brain barrier breakdown after SAH [48]. Our experiments provide direct evidence to show that CypA induced pericyte-associated blood-brain barrier disruption after SAH via the NF- $\mathrm{kB} / \mathrm{MMP} 9$ pathway.

Nevertheless, this study has limitations. The focus was on the CypA-CD147 pathway; thus, NF- $\kappa B$ translocation was not studied in detail. NF- $\mathrm{kB}$ translocation, which elevates MMP9 transcription, has been well established in other studies. In addition, CypA may be located in the cytoplasm and the extracellular region and could affect surrounding cells. Consistent with previous studies, we found that pericytes release CypA as an autocrine signaling molecule. However, whether other types of cells secrete CypA and have effects on pericytes after SAH are unknown. Other unknown mechanisms of CypA cannot be ruled out; thus, in our future studies, the expression of CypA in other cell types and the mechanisms of CypA will be clarified. 


\section{Conclusions}

This study demonstrated for the first time that CypA is elevated in the pericytes of the brain after $\mathrm{SAH}$, and extraneous recombinant CypA damages the blood-brain barrier and aggravates the neurological outcome. This process was potentially mediated by CD147, which may promote NF- $\mathrm{kB}$ nuclear translocation, MMP9 secretion, and junction protein degradation in the brain. By targeting CypA and pericytes, our present data and further translational studies may provide new insights on the management of SAH patients.

\section{Supplementary information}

Supplementary information accompanies this paper at https://doi.org/10. 1186/s12974-020-1699-6

Additional file 1: Figure S1. The experimental design of present study.

\begin{abstract}
Abbreviations
ANOVA: Analysis of variance; CPZ: Chlorpromazine; CypA: Cyclophilin A; EBI: Early brain injury; FBS: Fetal bovine serum; Hb: Oxyhemoglobin; KO: Knockout; PBS: Phosphate-buffered saline; SAH: Subarachnoid hemorrhage; Scr siRNA: Scrambled small interference RNA; siRNA: Small interference RNA; TJs: Tight junctions
\end{abstract}

\section{Acknowledgments}

Not applicable.

\section{Authors' contributions}

$Y C, H F$ and PP designed the experimental protocols. PP, HZ, XZ, QL, JQ, SZ, $\mathrm{FY}$, and $\mathrm{HH}$ performed the experiments. YC, PP, JHZ and $\mathrm{XL}$ carried out data analysis. YC, PP and HF prepared and revised the manuscript. YC and HF gave the final approval of manuscript to be published.

\section{Funding}

This work was supported by the Talent Support Program of Southwest Hospital (SWH2018BJKJ-05), the National Natural Science Foundation of China (Grant Nos. 81220108009), and the Natural Science Foundation of Liaoning province (Grant Nos. 20180550504).

\section{Availability of data and materials}

Data sharing is not applicable to this article as no datasets were generated or analyzed during the current study.

\section{Ethics approval and consent to participate}

All experimental procedures were approved by the Ethics Committee of Southwest Hospital and performed in accordance with the guidelines in the National Institutes of Health Guide for the Care and Use of Laboratory Animals and followed the ARRIVE guidelines (Animal Research: Reporting in Vivo Experiments, https://www.nc3rs.org.uk/arrive-guidelines).

\section{Consent for publication}

Not applicable.

\section{Competing interests}

The authors declare that they have no competing interests.

\section{Author details}

${ }^{1}$ Department of Neurosurgery, Southwest Hospital, Army Medical University (Third Military Medical University), Chongqing 400038, China. ${ }^{2}$ Department of Neurosurgery, General Hospital of Northern Theater Command (Shenyang Military Command), Shenyang 110016, China. ${ }^{3}$ Department of Pathophysiology and High-Altitude Pathology, College of High-Altitude Military Medicine, Third Military Medical University, Chongqing 400038, China. ${ }^{4}$ Neuroscience Research Center, Loma Linda University, Loma Linda, California 92350, USA. ${ }^{5}$ Institute of Immunology, Army Medical University
(Third Military Medical University), Chongqing 400038, China. ${ }^{6}$ State Key Laboratory of Trauma, Burn and Combined Injury, Third Military Medical University, Chongqing 400038, China. ${ }^{7}$ Chongqing Key Laboratory of Precision Neuromedicine and Neuroregenaration, Southwest Hospital, Third Medical University, Chongqing 400038, China.

Received: 2 November 2019 Accepted: 2 January 2020

Published online: 11 January 2020

\section{References}

1. Sehba FA, Hou J, Pluta RM, Zhang JH. The importance of early brain injury after subarachnoid hemorrhage. Prog Neurobiol. 2012;97:14-37.

2. Zhang JH, Badaut J, Tang JP, Obenaus A, Hartman R, Pearce WJ. The vascular neural network-a new paradigm in stroke pathophysiology. Nat Rev Neurol. 2012:8:711-6.

3. Armulik A, Genove G, Mae M, Nisancioglu MH, Wallgard E, Niaudet C, He LQ, Norlin J, Lindblom P, Strittmatter K, et al. Pericytes regulate the bloodbrain barrier. Nature. 2010;468:557-U231.

4. Peppiatt CM, Howarth C, Mobbs P, Attwell D. Bidirectional control of CNS capillary diameter by pericytes. Nature. 2006:443:700-4

5. Liu Z, Li Q, Cui G, Zhu G, Tang W, Zhao H, Zhang JH, Chen Y, Feng H. Blood-filled cerebrospinal fluid-enhanced pericyte microvasculature contraction in rat retina: a novel in vitro study of subarachnoid hemorrhage. Exp Ther Med. 2016:12:2411-6.

6. Li Q, Chen Y, Li B, Luo C, Zuo S, Liu X, Zhang JH, Ruan H, Feng H. Hemoglobin induced NO/cGMP suppression deteriorate microcirculation via pericyte phenotype transformation after subarachnoid hemorrhage in rats. Sci Rep. 2016:6:22070.

7. Bell RD, Winkler EA, Singh I, Sagare AP, Deane R, Wu Z, Holtzman DM, Betsholtz C, Armulik A, Sallstrom J, et al. Apolipoprotein E controls cerebrovascular integrity via cyclophilin A. Nature. 2012;485:512-6.

8. Underly RG, Levy M, Hartmann DA, Grant RI, Watson AN, Shih AY. Pericytes as inducers of rapid, matrix metalloproteinase-9-dependent capillary damage during ischemia. J Neurosci. 2017;37:129-40.

9. Chen $Y$, Li Q, Tang J, Feng H, Zhang JH. The evolving roles of pericyte in early brain injury after subarachnoid hemorrhage. Brain Res. 2015;1623:110-22.

10. Kahan BD. Cyclosporine. N Engl J Med. 1989;321:1725-38.

11. Pan P, Zhang X, Li Q, Zhao H, Qu J, Zhang JH, Liu X, Feng H, Chen Y. Cyclosporine A alleviated matrix metalloproteinase 9 associated blood-brain barrier disruption after subarachnoid hemorrhage in mice. Neurosci Lett. 2017:649:7-13.

12. Li B, Luo C, Tang W, Chen Z, Li Q, Hu B, Lin J, Zhu G, Zhang JH, Feng H. Role of HCN channels in neuronal hyperexcitability after subarachnoid hemorrhage in rats. J Neurosci. 2012:32:3164-75.

13. Chen Y, Zhang Y, Tang J, Liu F, Hu Q, Luo C, Tang J, Feng H, Zhang JH. Norrin protected blood-brain barrier via frizzled-4/beta-catenin pathway after subarachnoid hemorrhage in rats. Stroke. 2015;46:529-36.

14. Sugawara $T$, Ayer $R$, Jadhav $V$, Zhang JH. A new grading system evaluating bleeding scale in filament perforation subarachnoid hemorrhage rat model. J Neurosci Methods. 2008;167:327-34.

15. Engelhardt S, Huang SF, Patkar S, Gassmann M, Ogunshola OO. Differential responses of blood-brain barrier associated cells to hypoxia and ischemia: a comparative study. Fluids Barriers CNS. 2015;12:4.

16. Li B, He Y, Xu L, Hu Q, Tang J, Chen Y, Tang J, Feng H, Zhang JH. Progranulin reduced neuronal cell death by activation of Sortilin 1 signaling pathways after subarachnoid hemorrhage in rats. Crit Care Med. 2015;43: e304-11.

17. Zuo S, Ge H, Li Q, Zhang X, Hu R, Hu S, Liu X, Zhang JH, Chen Y, Feng H. Artesunate protected blood-brain barrier via Sphingosine 1 phosphate receptor 1/phosphatidylinositol 3 kinase pathway after subarachnoid hemorrhage in rats. Mol Neurobiol. 2017;54:1213-28.

18. Zhang Y, Chen Y, Wu J, Manaenko A, Yang P, Tang J, Fu W, Zhang JH. Activation of dopamine D2 receptor suppresses neuroinflammation through alphaB-crystalline by inhibition of NF-kappaB nuclear translocation in experimental ICH mice model. Stroke. 2015;46:2637-46.

19. Zhao Z, Nelson AR, Betsholtz C, Zlokovic BV. Establishment and dysfunction of the blood-brain barrier. Cell. 2015;163:1064-78.

20. Kut C, Grossman SA, Blakeley J. How critical is the blood-brain barrier to the development of neurotherapeutics? JAMA Neurol. 2015;72:381-2.

21. Obermeier B, Daneman R, Ransohoff RM. Development, maintenance and disruption of the blood-brain barrier. Nat Med. 2013;19:1584-96. 
22. Lai $\mathrm{CH}$, Kuo $\mathrm{KH}$. The critical component to establish in vitro BBB model: Pericyte. Brain Res Brain Res Rev. 2005;50:258-65.

23. Shimizu F, Sano Y, Maeda T, Abe MA, Nakayama H, Takahashi R, Ueda M, Ohtsuki S, Terasaki T, Obinata M, Kanda T. Peripheral nerve pericytes originating from the blood-nerve barrier expresses tight junctional molecules and transporters as barrier-forming cells. J Cell Physiol. 2008; 217:388-99.

24. Allt G, Lawrenson JG. Pericytes: cell biology and pathology. Cells Tissues Organs. 2001;169:1-11.

25. Sweeney MD, Ayyadurai S, Zlokovic BV. Pericytes of the neurovascular unit: key functions and signaling pathways. Nat Neurosci. 2016;19:771-83.

26. Machida T, Takata F, Matsumoto J, Takenoshita H, Kimura I, Yamauchi A, Dohgu S, Kataoka Y. Brain pericytes are the most thrombin-sensitive matrix metalloproteinase-9-releasing cell type constituting the blood-brain barrier in vitro. Neurosci Lett. 2015;599:109-14.

27. Nigro P, Pompilio G, Capogrossi MC. Cyclophilin A: a key player for human disease. Cell Death Dis. 2013;4:e888.

28. Bukrinsky M. Extracellular cyclophilins in health and disease. Biochim Biophys Acta. 1850;2015:2087-95.

29. Colgan J, Asmal M, Neagu M, Yu B, Schneidkraut J, Lee Y, Sokolskaja E, Andreotti A, Luban J. Cyclophilin A regulates TCR signal strength in CD4+ T cells via a proline-directed conformational switch in Itk. Immunity. 2004;21: 189-201.

30. Satoh K, Nigro P, Berk BC. Oxidative stress and vascular smooth muscle cell growth: a mechanistic linkage by cyclophilin A. Antioxid Redox Signal. 2010; 12:675-82.

31. Suzuki J, Jin ZG, Meoli DF, Matoba T, Berk BC. Cyclophilin A is secreted by a vesicular pathway in vascular smooth muscle cells. Circ Res. 2006;98:811-7.

32. Soe NN, Sowden M, Baskaran P, Kim Y, Nigro P, Smolock EM, Berk BC. Acetylation of cyclophilin $A$ is required for its secretion and vascular cell activation. Cardiovasc Res. 2014;101:444-53.

33. Dang B, Li H, Xu X, Shen H, Wang Y, Gao A, He W, Wang Z, Chen G. Cyclophilin A/cluster of differentiation 147 interactions participate in early brain injury after subarachnoid hemorrhage in rats. Crit Care Med. 2015;43: e369-81.

34. Zhan Y, Chen C, Suzuki H, Hu Q, Zhi X, Zhang JH. Hydrogen gas ameliorates oxidative stress in early brain injury after subarachnoid hemorrhage in rats. Crit Care Med. 2012;40:1291-6.

35. Feng Y, Yu S, Lasell TK, Jadhav AP, Macia E, Chardin P, Melancon P, Roth M, Mitchison T, Kirchhausen T. Exo1: a new chemical inhibitor of the exocytic pathway. Proc Natl Acad Sci U S A. 2003;100:6469-74.

36. Feng $Y$, Jadhav AP, Rodighiero C, Fujinaga $Y$, Kirchhausen T, Lencer WI. Retrograde transport of cholera toxin from the plasma membrane to the endoplasmic reticulum requires the trans-Golgi network but not the Golgi apparatus in Exo2-treated cells. EMBO Rep. 2004;5:596-601.

37. Carpentier M, Descamps L, Allain F, Denys A, Durieux S, Fenart L, Kieda C, Cecchelli R, Spik G. Receptor-mediated transcytosis of cyclophilin B through the blood-brain barrier. J Neurochem. 1999;73:260-70.

38. Woltman AM, Schlagwein N, van der Kooij SW, van Kooten C. The novel cyclophilin-binding drug sanglifehrin A specifically affects antigen uptake receptor expression and endocytic capacity of human dendritic cells. J Immunol. 2004;172:6482-9.

39. Hackstein H, Steinschulte C, Fiedel S, Eisele A, Rathke V, Stadlbauer T, Taner T, Thomson AW, Tillmanns H, Bein G, Holschermann H. Sanglifehrin a blocks key dendritic cell functions in vivo and promotes long-term allograft survival together with low-dose CsA. Am J Transplant. 2007;7:789-98.

40. Giardina BJ, Stein $\mathrm{K}$, Chiang HL. The endocytosis gene END3 is essential for the glucose-induced rapid decline of small vesicles in the extracellular fraction in Saccharomyces cerevisiae. J Extracell Vesicles. 2014;3.

41. Kaushik DK, Hahn JN, Yong WW. EMMPRIN, an upstream regulator of MMPs, in CNS biology. Matrix Biol. 2015;44-46:138-46.

42. Agrawal SM, Silva C, Tourtellotte WW, Yong WW. EMMPRIN: a novel regulator of leukocyte transmigration into the CNS in multiple sclerosis and experimental autoimmune encephalomyelitis. J Neurosci. 2011;31:669-77.

43. Agrawal SM, Silva C, Wang J, Tong JP, Yong WW. A novel anti-EMMPRIN function-blocking antibody reduces $T$ cell proliferation and neurotoxicity: relevance to multiple sclerosis. J Neuroinflammation. 2012;9:64.

44. Agrawal SM, Williamson J, Sharma R, Kebir H, Patel K, Prat A, Yong WW. Extracellular matrix metalloproteinase inducer shows active perivascular cuffs in multiple sclerosis. Brain. 2013;136:1760-77.
45. Agrawal SM, Yong W. The many faces of EMMPRIN — roles in neuroinflammation. Biochim Biophys Acta. 2011;1812:213-9.

46. Zhu W, Khachi S, Hao Q, Shen F, Young WL, Yang GY, Chen Y. Upregulation of EMMPRIN after permanent focal cerebral ischemia. Neurochem Int. 2008; 52:1086-91.

47. Boulos S, Meloni BP, Arthur PG, Majda B, Bojarski C, Knuckey NW. Evidence that intracellular cyclophilin A and cyclophilin A/CD147 receptor-mediated ERK1/2 signalling can protect neurons against in vitro oxidative and ischemic injury. Neurobiol Dis. 2007;25:54-64.

48. Chen S, Zhang J. Cyclophilin A/Cluster of Differentiation 147 Interactions and Blood-Brain Barrier Disruption After Subarachnoid Hemorrhage. Crit Care Med. 2015;43:e593-4.

\section{Publisher's Note}

Springer Nature remains neutral with regard to jurisdictional claims in published maps and institutional affiliations.
Ready to submit your research? Choose BMC and benefit from:

- fast, convenient online submission

- thorough peer review by experienced researchers in your field

- rapid publication on acceptance

- support for research data, including large and complex data types

- gold Open Access which fosters wider collaboration and increased citations

- maximum visibility for your research: over $100 \mathrm{M}$ website views per year

At BMC, research is always in progress.

Learn more biomedcentral.com/submissions 